\title{
A programmed cell death pathway in the malaria parasite Plasmodium falciparum has general features of mammalian apoptosis but is mediated by clan CA cysteine proteases
}

\author{
J-H Ch'ng ${ }^{1}$, SR Kotturi ${ }^{2}$, AG-L Chong ${ }^{3}$, MJ Lear ${ }^{2,4}$ and KS-W Tan ${ }^{\star, 1,3}$
}

\begin{abstract}
Several recent discoveries of the hallmark features of programmed cell death (PCD) in Plasmodium falciparum have presented the possibility of revealing novel targets for antimalarial therapy. Using a combination of cell-based assays, flow cytometry and fluorescence microscopy, we detected features including mitochondrial dysregulation, activation of cysteine proteases and in situ DNA fragmentation in parasites induced with chloroquine (CQ) and staurosporine (ST). The use of the pan-caspase inhibitor, z-Val-Ala-Asp-fmk (zVAD), and the mitochondria outer membrane permeabilization (MOMP) inhibitor, 4-hydroxytamoxifen, enabled the characterization of a novel $C Q$-induced pathway linking cysteine protease activation to downstream mitochondrial dysregulation, amplified protease activity and DNA fragmentation. The PCD features were observed only at high $(\mu \mathrm{M})$ concentrations of $\mathrm{CQ}$. The use of a new synthetic coumarin-labeled chloroquine (CM-CQ) showed that these features may be associated with concentration-dependent differences in drug localization. By further using cysteine protease inhibitors z-AspGlu-Val-Asp-fmk (zDEVD), z-Phe-Ala-fmk (zFA), z-Phe-Phe-fmk (zFF), z-Leu-Leu-Leu-fmk (zLLL), E64d and CA-074, we were able to implicate clan CA cysteine proteases in CQ-mediated PCD. Finally, CQ induction of two CQ-resistant parasite strains, 7G8 and K1, reveals the existence of PCD features in these parasites, the extent of which was less than 3D7. The use of the chemoreversal agent verapamil implicates the parasite digestive vacuole in mediating CQ-induced PCD.
\end{abstract}

Cell Death and Disease (2010) 1, e26; doi:10.1038/cddis.2010.2; published online 18 February 2010

Subject Category: Experimental Medicine

This is an open-access article distributed under the terms of the Creative Commons Attribution License, which permits distribution and reproduction in any medium, provided the original author and source are credited. This license does not permit commercial exploitation without specific permission.

The most deadly of the four clinically relevant malaria parasites, Plasmodium falciparum, continues to kill an estimated one million people annually. ${ }^{1}$ Although current drugs can combat the disease, the increase in resistance to these medicines makes it crucial for new leads to be uncovered, such that new therapeutic initiatives will be one step ahead of disease progression. ${ }^{2}$ One pathway yet to be thoroughly explored for antimalarial development is that of programmed cell death (PCD).

Recent reports highlight the existence of PCD features in unicellular organisms including Blastocystis, Leishmania, trypanosomes, Saccharomyces cerevisiae and Trichomonas vaginalis. $^{3}$ Indeed, accounts of $\mathrm{PCD}$ in $P$. falciparum ${ }^{4}$ and the rodent malaria parasite $P$. berghe ${ }^{5}$ have been documented, as featured by the fragmentation of DNA and a loss of mitochondria membrane potential. However, other reports suggest that $P$. falciparum does not undergo PCD. ${ }^{6-7}$

Besides inconclusive cell death features in the malaria pathogen, there is a lack of evidence for a clear pathway linking such features. In addition, PCD pathway mediators have yet to be identified. Some studies have made use of caspase inhibitors or substrates to suggest the involvement of clan CD cysteine protease mediators such as metacaspaseorthologs $^{8}$ or caspase 3-like proteases. ${ }^{9}$ These assays, however, have been shown to be nonspecific and do not reliably discriminate between the clan $C A$ and $C D$ cysteine proteases. $^{10-11}$

Our study began by characterizing the PCD pathway of $P$. falciparum. First, we conducted three cell-based assays and detected mitochondrial dysregulation, cysteine protease activation and DNA fragmentation in both CQ- and

\footnotetext{
${ }^{1}$ Department of Microbiology, Yong Loo Lin School of Medicine, National University of Singapore, Singapore; ${ }^{2}$ Department of Chemistry, National University of Singapore, Singapore; ${ }^{3}$ Infectious Disease Program, Life Sciences Institute, National University of Singapore, Singapore and ${ }^{4}$ Faculty of Science, Medicinal Chemistry Program, Life Sciences Institute, National University of Singapore, Singapore

${ }^{*}$ Corresponding author: KS-W Tan, Department of Microbiology, National University of Singapore, 5 Science Drive 2, Singapore 117597, Singapore.

Tel: + 656516 6780; Fax: + 656776 6872; E-mail: mictank@nus.edu.sg

Keywords: malaria; Plasmodium falciparum; programmed cell death; clan CA cysteine protease; chloroquine; verapamil

Abbreviations: $\mathrm{PCD}$, programmed cell death; $\mathrm{CQ}$, chloroquine; $\mathrm{CM}$, coumarin, i.e., 7-dimethylamino-4-coumarinacetic acid; $\mathrm{CM}-\mathrm{CQ}$, coumarin-labeled chloroquine; ST, staurosporine; MOMP, mitochondria outer membrane permeabilization; $\Delta \psi_{\mathrm{m}}$, mitochondrial transmembrane potential; 4HT, 4-hydroxy-tamoxifen; TUNEL, terminal deoxynucleotidyltransferase dUTP nicked-end labeling; fmk, fluoromethylketone; VP, verapamil

Received 20.11.09; revised 11.12.09; accepted 17.12.09; Edited by D Bano
} 
ST-induced parasites. Second, the mitochondria outer membrane permeabilization (MOMP) inhibitor, 4-hydroxytamoxifen, and pan-caspase inhibitor, z-Val-Ala-Asp-fmk (zVAD), were used to determine the order in which these PCD features occurred. Interestingly, the sequence of events in CQ-induced parasites showed a striking resemblance to the classical mammalian apoptotic pathway even in the absence of classical apoptotic machinery. Third, owing to the crossreactive nature of fluoromethylketone (fmk) inhibitors, clan CA-specific protease inhibitors E64d and CA-074 were used and showed that clan CA cysteine proteases are crucial mediators in the $C Q$-induced $P C D$ pathway. Clan $C A$ proteases, in particular the cathepsins and calpains, have already been implicated in protozoan PCD. ${ }^{12-15}$ As such, the discovery of clan CA proteases as PCD mediators in $P$. falciparum presents a clear new target of therapeutic intervention for future antimalarial design. Finally, the existence of CQ-induced PCD was shown in drug-resistant strains $7 \mathrm{G} 8$ and $\mathrm{K} 1$, and the importance of CQ's lysosomotropic nature in triggering these features is discussed.

\section{Results}

Mitochondrial membrane potential assay. The accumulation of JC-1 J-aggregates in uninduced cells showed that about $90 \%$ of the parasites contained functional mitochondrion with transmembrane potential $\left(\Delta \psi_{\mathrm{m}}\right)$ (Figure 1a and $\mathrm{c}$ ). Confocal microscopy confirmed the specificity of the aggregated mitochondrial stain (Figure 1b). Treatment with CQ or ST caused a significant decline in this proportion to approximately 69 and $75 \%$, respectively (Figure 1a).

Pretreatment of parasite culture with 4-hydroxy-tamoxifen (4HT) inhibited CQ-induced MOMP almost entirely, returning the proportion of JC1-positive cells to $\sim 90 \%$ (Figure 1a). However, $4 \mathrm{HT}$ was unable to exert a similar protective effect for ST-induced parasites, suggesting that ST-induced decrease in JC-1 values was independent of MOMP.

Pan-caspase inhibitor, zVAD, was similarly able to rescue $\mathrm{CQ}$-induced cells from loss of $\Delta \psi_{\mathrm{m}}$ but was unable to protect cells from ST-induced JC-1 loss (Figure 1a). Almost $90 \%$ of $\mathrm{CQ}$-induced cells pretreated with ZVAD were JC1-positive compared with $77 \%$ of ST-induced cells. This suggests that the activity of cysteine proteases is upstream of MOMP in $\mathrm{CQ}$-induced cells.

The use of $4 \mathrm{HT}$ and $\mathrm{zVAD}$ in combination did not rescue ST-induced cells from JC-1 loss, whereas CQ-induced cells were completely rescued (Figure 1a).

Cysteine protease activity. In parasitized erythrocytes, high background staining within the erythrocyte (data not shown) deemed it necessary for parasites to be enriched by saponin-lysis of the host erythrocyte such that fluorescence of the parasite itself could be clearly recorded. In uninduced parasites, a basal level of CaspaTag activity was detected in $10 \%$ of the cells (Figure $2 a$ and $\mathrm{c}$ ). This proportion increased significantly on $\mathrm{CQ}$ and ST induction to 34 and $32 \%$, respectively. The retention of CaspaTag in a greater proportion of CQ- and ST-induced parasites suggested that the pan-caspase stain was bound to cysteine proteases that had been activated by the drug treatments.

Preincubation of the parasite culture with $z V A D$ reduced the basal proportion of uninduced cells that are CaspaTagpositive to $5 \%$ and suppressed CQ-induced CaspaTag binding to below 10\% (Figure 2a). Interestingly, zVAD did not reduce the proportion of CaspaTag-positive cells after ST treatment.

Even in the absence of $\mathrm{CQ}$ and ST, $4 \mathrm{HT}$ increased the proportion of CaspaTag-positive cells slightly to $17 \%$ (Figure 2a). Moreover, 4HT increased the proportion of CaspaTag-positive cells in ST-treated cultures from 32 to $80 \%$, suggesting synergism between $4 \mathrm{HT}$ and ST on the parasite. This synergistic effect was not preventable by zVAD treatment. Despite this inherent toxicity, 4HT reduced CaspaTag binding in CQ-induced cells from 34 to $15 \%$, showing clearly that MOMP is essential for cysteine protease activity in CQ-induced parasites.

In situ DNA fragmentation. As with the CaspaTag assay, parasitized cultures treated with various conditions were saponin-enriched just before TUNEL staining and gated in an identical manner. There was an increase in the proportion of TUNEL-positive cells from 10 to $27 \%$ and $56 \%$ in parasites induced by $C Q$ and ST, respectively, indicating that these two drugs induced in situ DNA fragmentation in parasites (Figure $3 a$ and $c$ ).

Pretreatment of parasite cultures with $4 \mathrm{HT}$ alone resulted in an increase in TUNEL-positive cells to $22.5 \%$ (Figure $3 a$ ). Moreover, $4 \mathrm{HT}$ was ineffective in reducing the percentage of TUNEL-positive cells in both CQ- and ST-treated cultures, possibly owing to its inherent toxicity.

Pretreatment with zVAD, however, significantly reduced the TUNEL-positive population in CQ-induced but not in ST-induced cultures (Figure 3a). This suggested that zVAD was able to effectively inhibit the pathway leading to in situ DNA fragmentation in CQ-induced parasites but not in STinduced populations.

In double inhibition studies, the proportion of CQ-induced TUNEL-positive cells decreased but the toxic effects of $4 \mathrm{HT}$ were still observable in the non-induced cultures (Figure 3a). ST-induced cultures remained highly TUNEL-positive even when inhibitors were used in combination.

Figure 1 JC-1 staining of infected RBCs shows loss of mitochondrial membrane potential $\left(\Delta \psi_{\mathrm{m}}\right)$ in Plasmodium falciparum parasites treated with drugs. (a) The proportion of JC1-positive parasites are represented in a bar chart to show populations pretreated with vehicle control, $10 \mu \mathrm{M}$ 4-hydroxytamoxifen (4HT) or $50 \mu \mathrm{M} \mathrm{z-VAD-fmk} \mathrm{(zVAD)} \mathrm{and}$ subjected to 8-h treatment with vehicle control $\square, 30 \mu \mathrm{M}$ chloroquine $\square$ or $10 \mu \mathrm{M}$ of staurosporine $\square$. Data represent means $\pm \mathrm{S}$.E. (AvB, AvC and BvF, P<0.001; BvE, $P<0.01 ; B v D, P<0.05 ; N=4)$ In each experiment, the full set of treatment conditions were carried out concurrently with the same batch of cells. Percentages represent ratios normalized to the healthy control population in each experiment. (b) Confocal microscopy of an uninfected erythrocyte and a trophozoite-infected erythrocyte (adjoining panels) with JC-1 monomer (green) accumulation in the parasite cytoplasm and J-aggregate (red) in the rod-shaped mitochondria of the same cell. (c) Flow cytometry histograms showing the gating of $\mathrm{JC} 1$ (red)-positive and $\mathrm{JC} 1$ (green)-positive populations, the ratio of which represents the JC1-positive population 
a

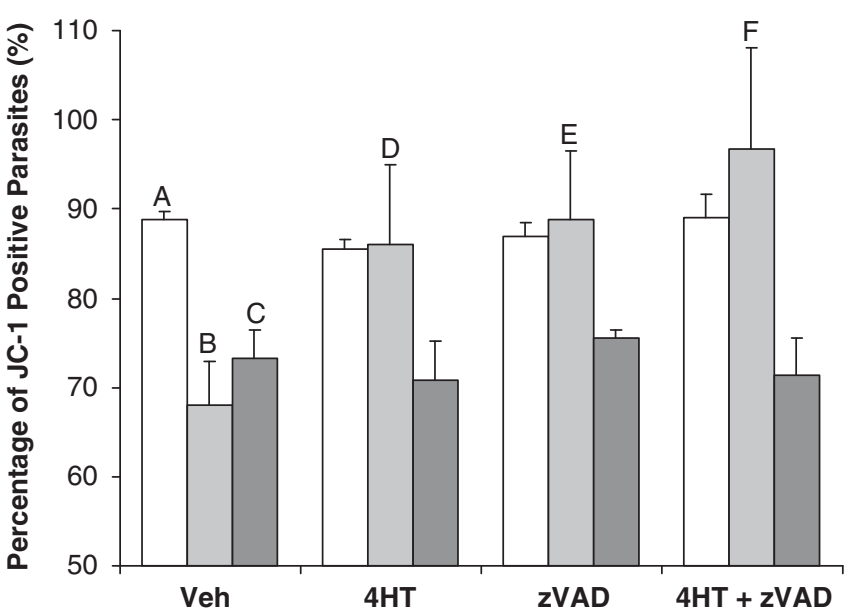

b

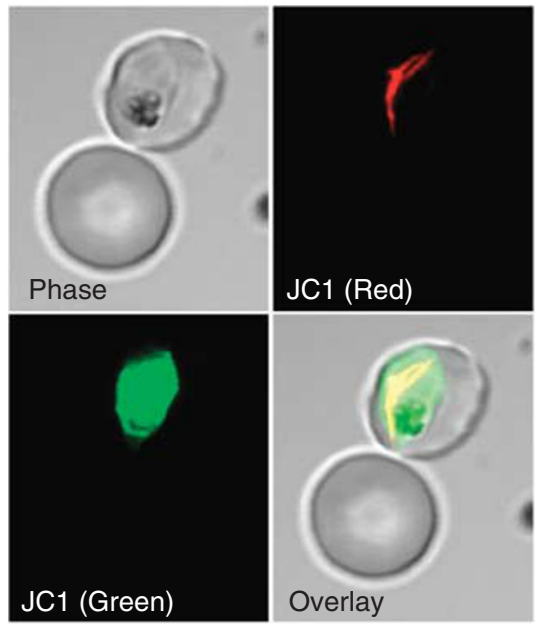

c
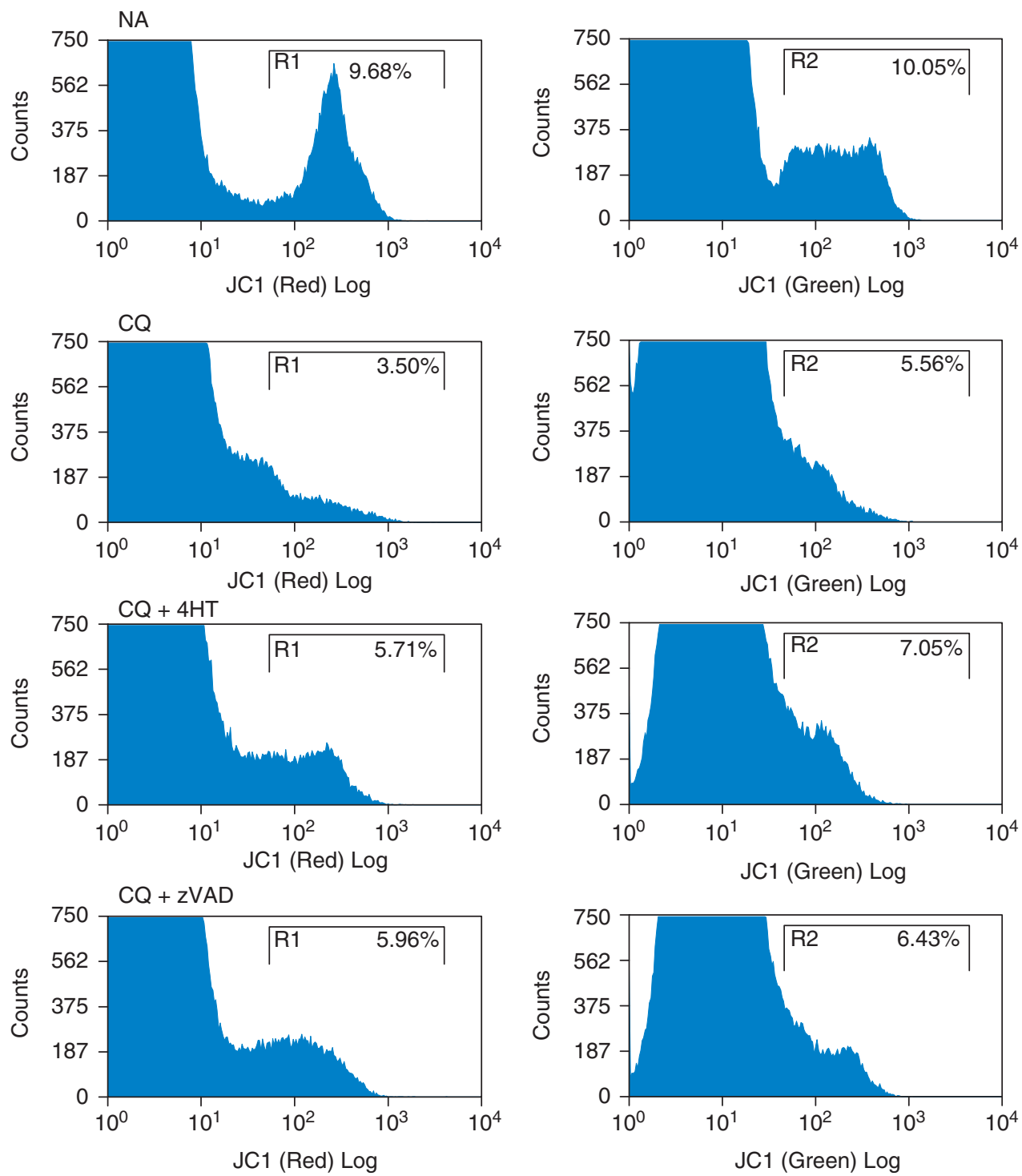

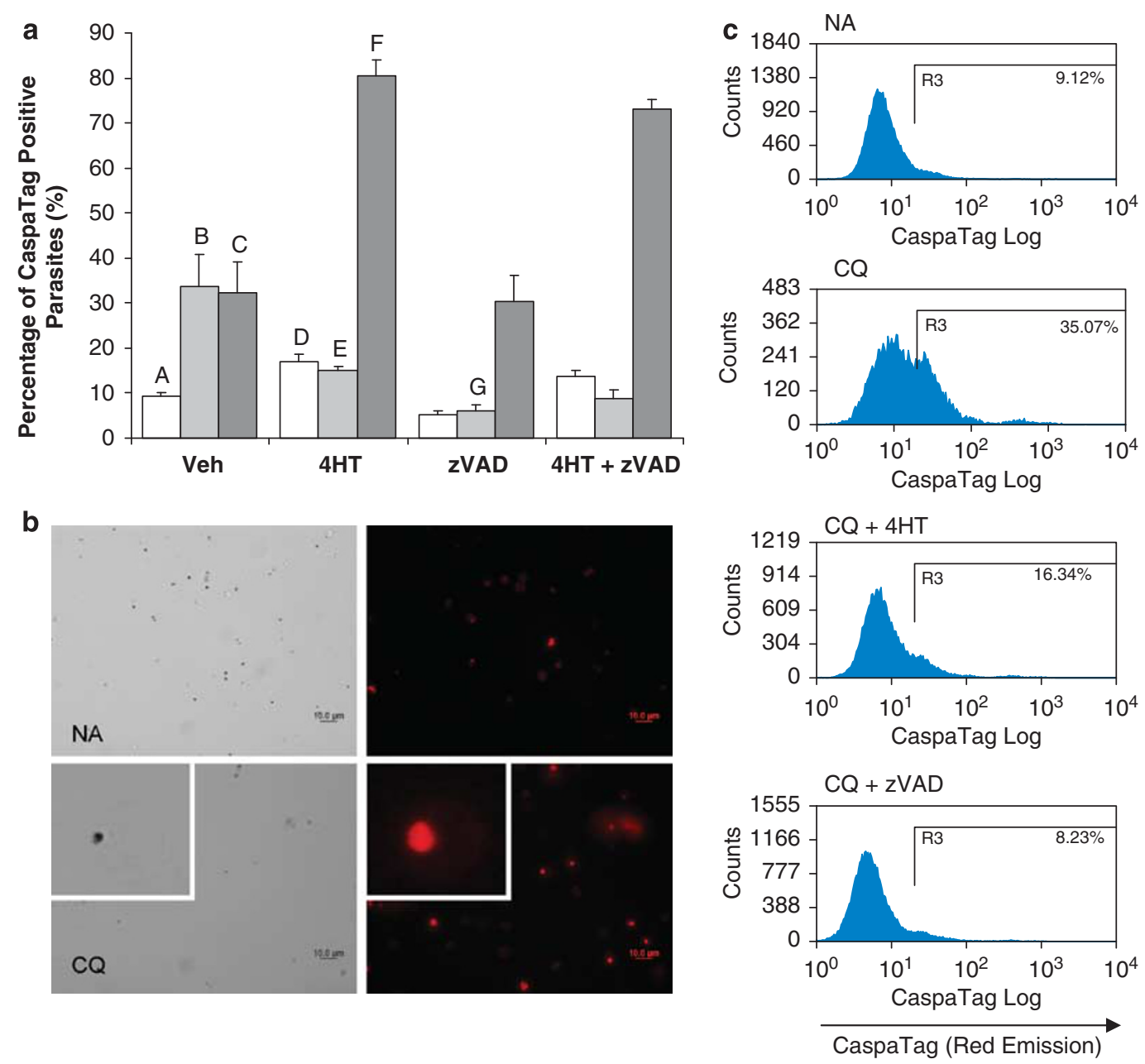

Figure 2 CaspaTag staining of saponin-enriched Plasmodium falciparum parasites shows the activation of cysteine proteases after incubation with drugs. (a) Percentage of CaspaTag-positive cells pretreated with vehicle control, $10 \mu \mathrm{M}$ 4-hydroxytamoxifen (4HT) or $50 \mu \mathrm{M} \mathrm{z-VAD-fmk} \mathrm{(zVAD)} \mathrm{and} \mathrm{subjected} \mathrm{to} 8 \mathrm{~h}$ treatment with vehicle control $\square, 30 \mu \mathrm{M}$ chloroquine $\square$ or $10 \mu \mathrm{M}$ of staurosporine $\square$. In each experiment, the full set of treatment conditions were carried out concurrently with the same batch of cells. Data represent means \pm S.E. (AvB, AvC, BvG, AvD, CvF and BvE, $P<0.001 ; N=3)(\mathbf{b})$ Fluorescent microscopy and (c) flow cytometry histograms show the increase in proportion of CaspaTag-positive cells after chloroquine treatment, with and without 30 min preincubation with ZVAD or 4HT

As CQ-induced parasites displayed clearer PCD induction and inhibition in MOMP, cysteine protease activity and in situ DNA fragmentation assays, we focused on the characterization of the CQ-induced pathway and its mediators.

Time course experiments with CaspaTag and JC-1. Time course experiments on $\mathrm{CQ}$-induced parasites showed that a significant decrease in JC-1-positive cells occurred after $4 \mathrm{~h}$ of drug administration in contrast to the increase in CaspaTag-positive cells that was significant only after $6 \mathrm{~h}$ (Figure 4). This suggests that mitochondrial dysregulation is an early-onset feature of PCD, which precedes the activation of cysteine proteases. As it was also shown that zVAD is able to prevent MOMP, the activity of cysteine proteases must be essential for the loss of $\Delta \psi_{\mathrm{m}}$ to take place. We therefore posit that a small increase in protease activity leads to mitochondrial permeabilization, which then amplifies the protease cascade in a positive feedback loop.
Preparation and validation of fluorescent-labeled chloroquine. To determine the localization of chloroquine (CQ), we synthesized a $C Q$ analog conjugated to biologically benign coumarin (CM) fluorophore. ${ }^{16}$ HPLC and NMR analyses indicated that coumarin-labeled chloroquine (CM-CQ) was synthesized with excellent purity of $98 \%$ (Supplementary data, Figure S11-13). To ascertain the biological efficacy of $\mathrm{CM}-\mathrm{CQ}, \mathrm{IC}_{50}$ curves were measured and the inhibitory efficiencies of the $C Q$ compounds determined (Supplementary data, Figure S1A). In addition, the effect of varying $\mathrm{CM}-\mathrm{CQ}$ concentrations on JC-1 assay was also measured (Supplementary data, Figure S2). These assays show that CM-CQ behaves similar to $C Q$ in parasite killing and inducing MOMP, but with about 10-fold less efficacy.

Dose-dependent effect of CQ. JC-1 and CaspaTag assays were used to investigate PCD features at different 
a

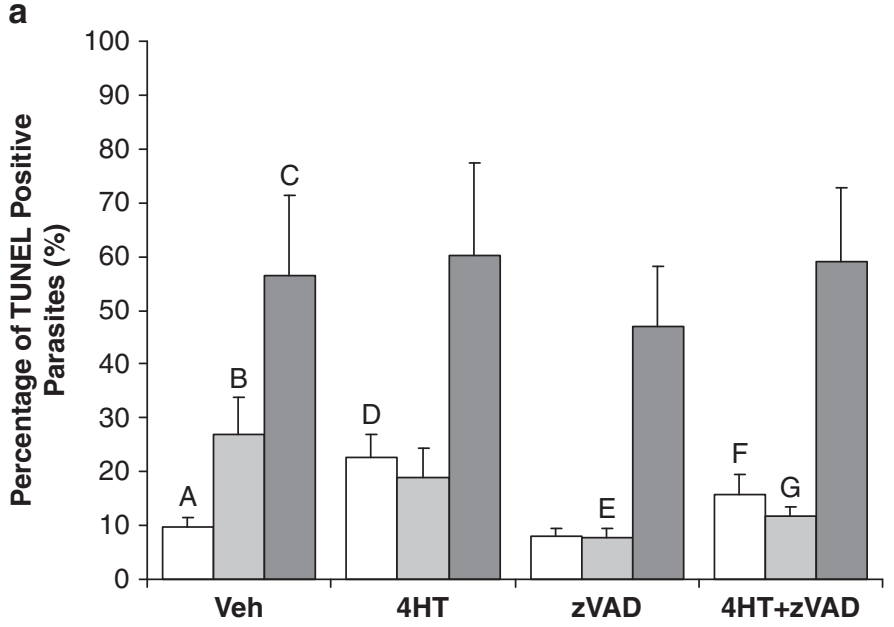

b NA

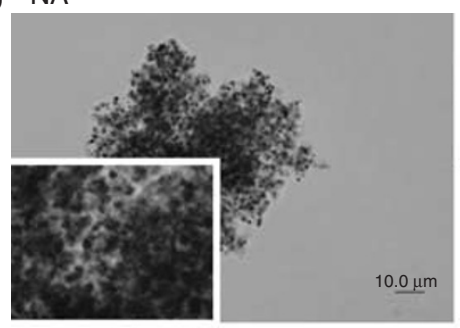

$\mathrm{CQ}$

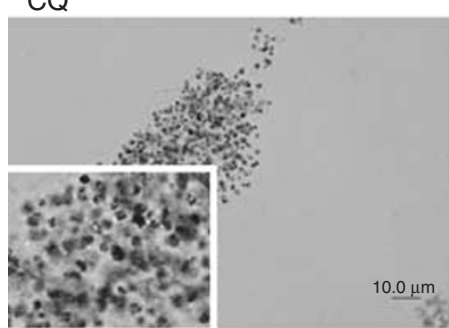

ST

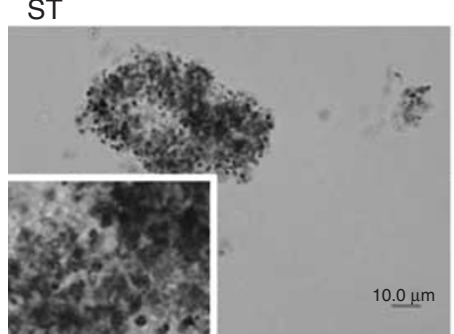

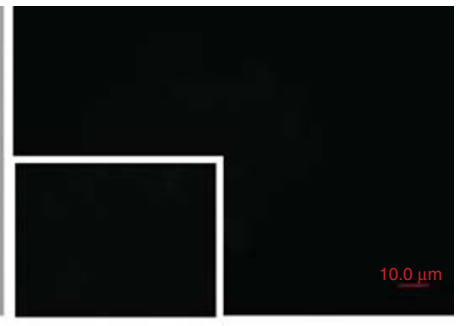
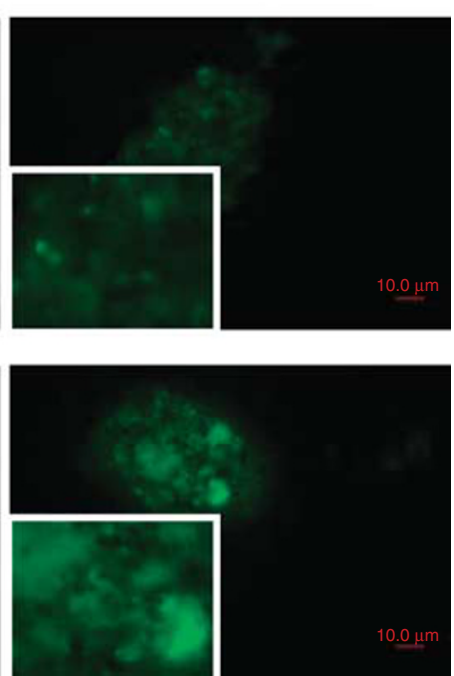
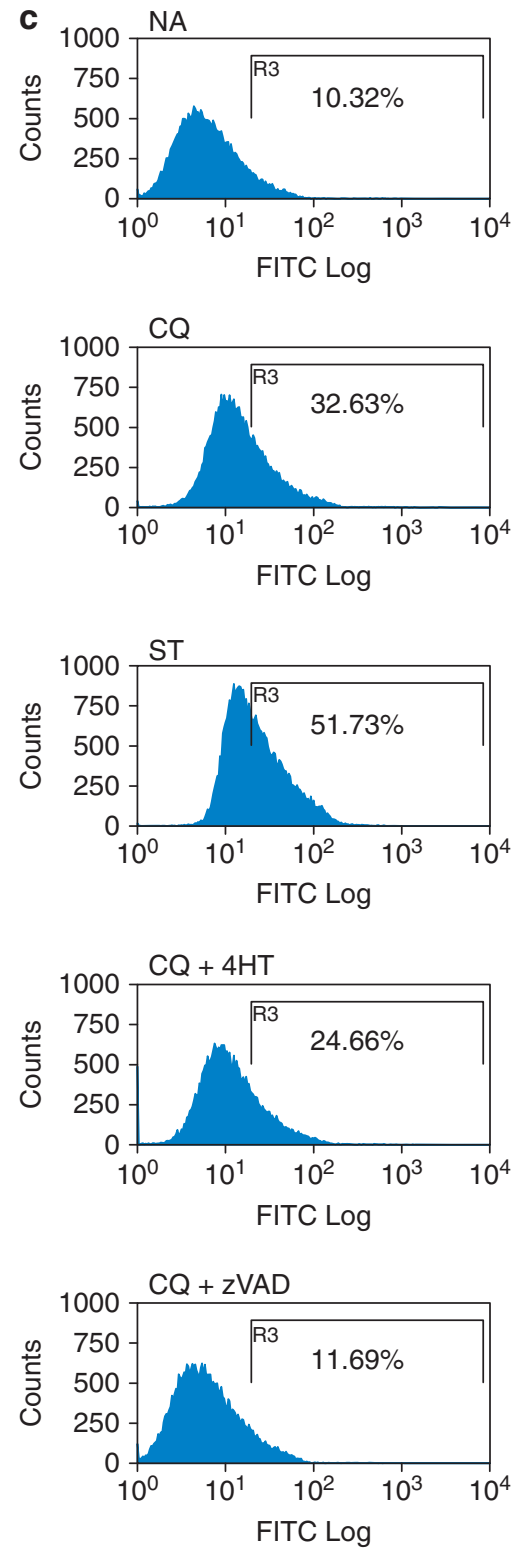

Figure 3 TUNEL staining of saponin-enriched Plasmodium falciparum parasites shows in situ DNA fragmentation occurring after incubation with drugs. (a) Percentage of TUNEL-positive cells pretreated with vehicle control, $10 \mu \mathrm{M}$ 4-hydroxytamoxifen (4HT) or $50 \mu \mathrm{M} \mathrm{z-VAD-fmk} \mathrm{(ZVAD),} \mathrm{subject} \mathrm{to} 8 \mathrm{~h}$ treatment with vehicle control $\square$, $30 \mu \mathrm{M}$ chloroquine $\square$ or $10 \mu \mathrm{M}$ of staurosporine $\square$. In each experiment, the full set of treatment conditions were carried out concurrently with the same batch of cells. Data represent means \pm S.E. (AvC, $P<0.001 ; A v B, A v D$ and BvE, $P<0.01 ; B v G, P<0.05 ; A v F, P=0.073 ; N=3$ ) (b) Fluorescent microscopy and (c) flow cytometry histograms show the increase in proportion of TUNEL-positive cells after $C Q$ and ST treatments, with and without 30 min preincubation with ZVAD and/or $4 H T$. For TUNEL-labeled cells, the quick fading of fluorescence deemed it necessary for cell clusters to be observed instead of single-cell suspensions

concentrations of $\mathrm{CQ}$ (Figure 5a). At low nanomolar concentrations, there were basal levels of PCD features but at micromolar concentrations of $C Q$, there was a large increase in the proportion of cells displaying MOMP and activation of cysteine proteases. Confocal microscopy revealed a dose-dependent relocalization of labeled $C Q$ from the DV at nanomolar concentrations to the cytoplasm at micromolar concentrations (Figure $5 \mathrm{~b}$ and Supplementary Data, Figure S3).

Necrosis control experiments. To exclude the possibility that the $C Q$-induced PCD is an unregulated necrotic death, parasitized cultures were subjected to sodium azide and $50^{\circ} \mathrm{C}$ treatments in the presence or absence of $\mathrm{zVAD}$ 


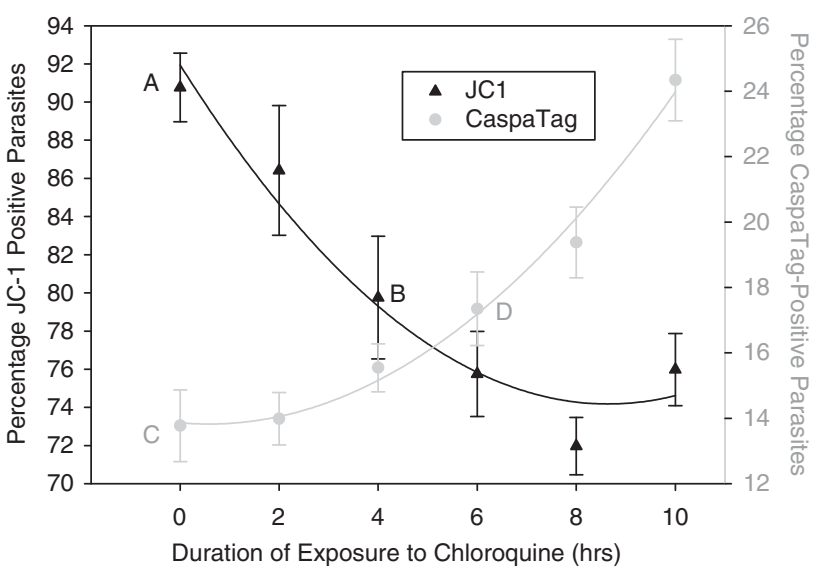

Figure 4 Time course study on chloroquine's effect on JC-1 (trend line in black) and CaspaTag (trend line in gray) staining of parasites. Chloroquine was added to parasite cultures every $2 \mathrm{~h}$ for $10 \mathrm{~h}$ and cells were assayed for the extent of mitochondrial transmembrane potential loss and activation of cysteine proteases. Data represent means \pm S.E. (AvB, $P<0.01 ; \mathrm{CvD}, P<0.05 ; N=2$ )

(Figure S4-S6). Both treatments resulted in a different cell death profile from the CQ-induced pathway and ZVAD was unable to inhibit these features. Heat treatment altered JC-1 background staining of the erythrocytes and therefore data were excluded.

Effect of different inhibitors on CaspaTag binding. Owing to the well-documented nonspecific binding of zVAD to both clan CD and CA proteases ${ }^{10-11,14,17}$ coupled with the absence of any caspase-orthologs in Plasmodium, ${ }^{18}$ the range of inhibitors was extended to elucidate the preferred substrate specificity of the target protease. Besides CA-074, all the tested inhibitors prevented the increase of CaspaTag-positive cells in CQ-induced samples (Figure 6a). Inhibition by clan CA-specific inhibitor E64d showed that target proteases were clan CA cysteine proteases. Inhibition by z-Phe-Ala-fmk (zFA), z-Phe-Phe-fmk (zFF) and z-Leu-Leu-Leu-fmk (zLLL) showed that target proteases lack the $\mathrm{P} 1$-aspartate specificity characteristic of caspases, and suggests the involvement of clan CA proteases such as cathepsins and calpain-like proteases in the pathway. Cathepsin B-specific CA-074 was unable to fully prevent CaspaTag binding, suggesting that cathepsin $\mathrm{B}$-like proteases are partly involved in mediating the pathway.

Hoechst staining of DNA content. To verify the effectiveness of the extended panel of inhibitors, parasites were assayed for a loss of DNA content $10 \mathrm{~h}$ after CQ treatment. Healthy ring-stage parasites were used to distinguish the G1 population (Supplementary data, Figure S7A) and a control experiment excluded the possibility that $C Q$ competed with Hoechst DNA-binding (Supplementary data, Figure $\mathrm{S} 7 \mathrm{C})$. Finally, flow cytometry histograms and fluorescence microscopy images (Supplementary data, Figure S7B and D) were compared to characterize parasite cultures that had been subject to various experimental conditions.

CQ-treated parasites showed an increased proportion of cells with sub-G1 DNA content from 10 to over $20 \%$ (Figure 6b). The effect of different inhibitors corroborated findings of the previous section, suggesting that clan CA proteases are essential in mediators for DNA degradation in CQ-induced PCD and that cathepsin B-like proteases act in concert with other clan CA mediators to give rise to DNA breakdown.

Intracellular calcium studies. In healthy parasites, calcium indicator fluo-4-AM localized to the digestive vacuole (DV) of healthy parasites (Supplementary data, Figure S8). However, $C Q$ treatment resulted in a redistribution of $\mathrm{Ca}^{2+}$ to the cytoplasm in a dose-dependent manner (Supplementary data, Figure S8 and S9). To explore the possibility that MOMP is $\mathrm{Ca}^{2+}$-mediated, a cell-permeable calcium chelator, BAPTA-AM, was used but this did not significantly reduce the proportion of $C Q$-induced parasites undergoing MOMP (Supplementary data, Figure S10).

Inter-strain comparisons for JC1 and Hoechst loss. We subsequently assayed for PCD features in CQ-resistant 7G8 and $\mathrm{K} 1$ strains (Figure 7). The $\mathrm{IC}_{50}$ values of $\mathrm{CQ}$ in $7 \mathrm{G} 8$ and $\mathrm{K} 1$ parasites were determined to be 76 and $202 \mathrm{nM}$, respectively (Supplementary Data, Figure S1B and S1C). Pretreatment with VP lowered the $\mathrm{IC}_{50}$ values of $7 \mathrm{G} 8$ and $\mathrm{K} 1$ to 61 and $120 \mathrm{nM}$, respectively. Interestingly, 7G8 cultures mirrored 3D7 and showed a significant drop in the JC1positive population at concentrations of $\mathrm{CQ}$ exceeding $3 \mu \mathrm{M}$ of $\mathrm{CQ}$ (Figure 7a). In comparison, the drop in JC1-positive K1 cells was markedly less at this same concentration, and it was only at a higher concentration of the drug that there was a significant decrease in the proportion of JC1-positive cells. However, pretreatment of $\mathrm{K} 1$ with $10 \mu \mathrm{M}$ of chemoreversal agent VP resulted in a significant decrease in JC1-positive cells even at $3 \mu \mathrm{M}$ of $\mathrm{CQ}$.

As with 3D7, both $7 \mathrm{G} 8$ and $\mathrm{K} 1$ showed a significant increase in the proportion of parasites with sub-G1 DNA content after $\mathrm{CQ}$ treatment (Figure $7 \mathrm{~b}$ ). However, these proportions were significantly lower than those observed in 3D7. Although VP pretreatment was able to significantly increase the proportion of $\mathrm{K} 1$ parasites with sub-G1 DNA content, this reversion was incomplete. It should be noted that BD LSRII showed better resolution of Hoechst-positive parasites from the uninfected RBC population, and consequently the proportion of cells showing DNA loss as measured by this cytometer was slightly greater than that measured in the previous set of experiments using DAKO cytomation.

\section{Discussion}

Our results show that both $C Q$ and $S T$ treatment gives rise to features of PCD in Plasmodium including MOMP, the activation of cysteine proteases and in situ DNA fragmentation. These findings corroborate growing evidence supporting the existence of PCD hallmarks in parasites induced by a variety of agents including drugs, ${ }^{8,19}$ febrile temperatures, ${ }^{19-20}$ exposure to bilirubin resulting in reactive oxygen species increase ${ }^{9}$ and binding of platelets to infected erythrocytes. ${ }^{21}$

Observation of apoptotic features such as chromatin condensation, DNA fragmentation, phosphatidylserine exposure, apoptotic body formation and caspase activity have been shown by Al-Olayan et al. ${ }^{5}$ in the mosquito midgut stage 
Effect of CQ Concentrations on JC1 and CaspaTag

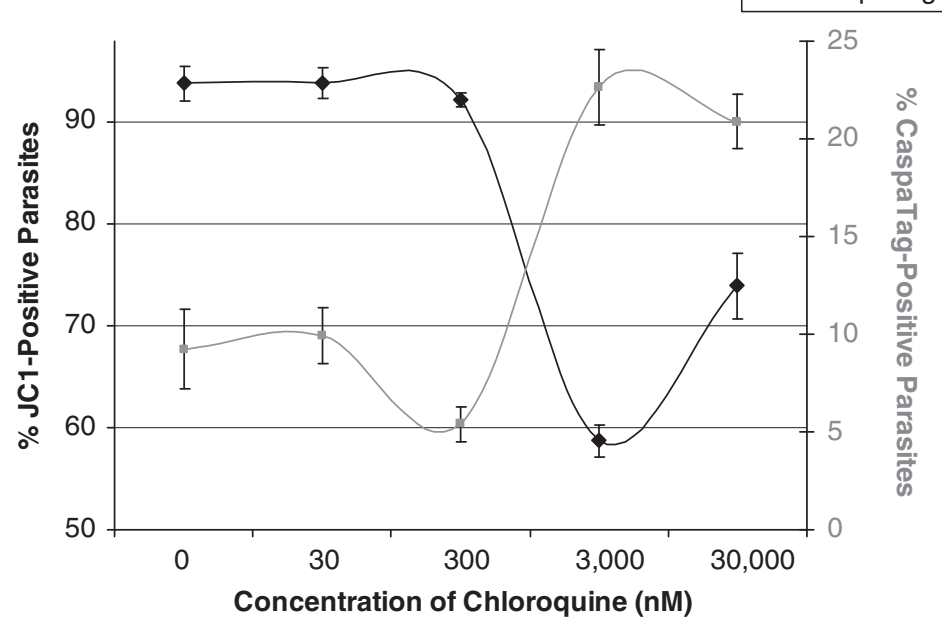

b
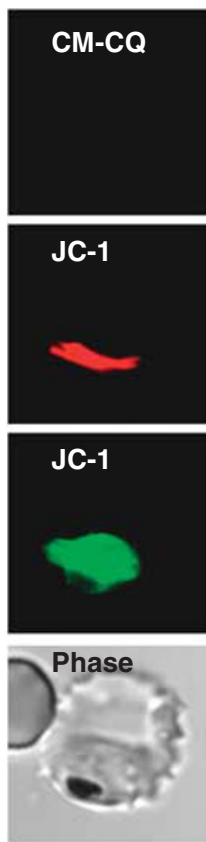

0 nM
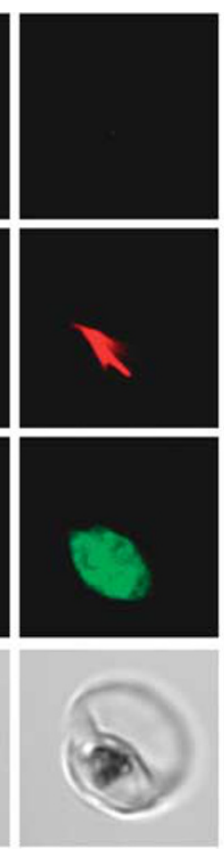

$30 \mathrm{nM}$
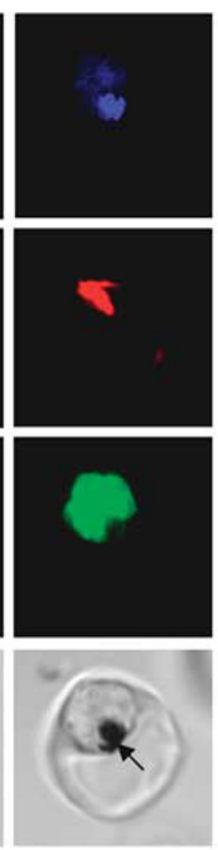

$300 \mathrm{nM}$
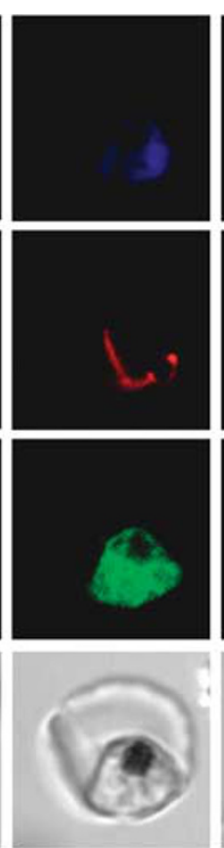

$3 \mu \mathbf{M}$
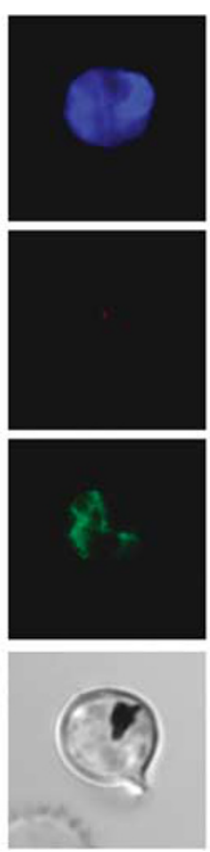

$30 \mu \mathrm{M}$
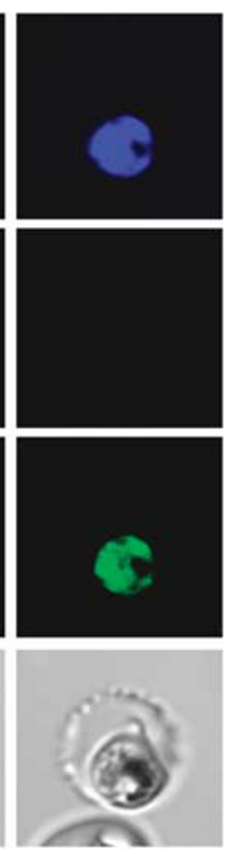

$300 \mu \mathrm{M}$

Concentration of Coumarin-labeled Chloroquine (CM-CQ)

Figure 5 (a) Dose-dependent study of chloroquine's effect on JC-1 loss (black line) and CaspaTag staining (gray line). Parasitized cultures were incubated for $8 \mathrm{~h}$ with different concentrations of chloroquine $(N=2)$. (b) Confocal microscopy shows JC-1 staining the mitochondrion of a healthy parasite (red) and its cytoplasm (green). No accumulation of coumarin-labeled chloroquine (CM-CQ) is detected at $30 \mathrm{nM}$, but at $300 \mathrm{nM}, \mathrm{CM}-\mathrm{CQ}$ localizes in the hemazoin-containing food vacuole (black arrow) of the parasite. Accumulation of $\mathrm{CM}-\mathrm{CQ}$ within the parasite is nonspecific at concentrations exceeding $3 \mu \mathrm{M}$ and localizes throughout the parasite's cytoplasm. At these concentrations, CM-CQ also induced mitochondria-outer membrane permeabilization resulting in mitochondria that were not so clearly defined by JC1 staining

of $P$. berghei. However, this could not be reproduced by Le Chat et al. $^{22}$ In apoptotic $P$. falciparum, as induced by drugs and heat-shock treatments, Nyakeriga et al. ${ }^{6}$ and Totino et al. ${ }^{7}$ were unable to detect MOMP, DNA fragmentation and laddering, or chromatin cleavage. In contrast, Meslin et al. ${ }^{8}$ and Kumar et al. ${ }^{9}$ showed MOMP, DNA fragmentation, chromatin condensation and DEVDase activity in other strains of $P$. falciparum induced by similar stimuli. Still other authors observed evidence of autophagy or secondary necrosis including cytoplasmic vacuolation in drug-resistant strains such as PSS1 or a swelling of the food vacuole in CSC-1.7,19 These discrepancies may be due to differing drug concentrations, parasite strains, developmental stages, inducers and assaying methods, complicating the conclusions that can be reliably discerned.

Another limitation of the existing studies has been the absence of definitive evidence outlining any pathway of PCD. As unicellular eukaryotes may display more than one PCD pathway, ${ }^{23}$ the focus of this study was primarily to delineate the pathway induced by $C Q$. 


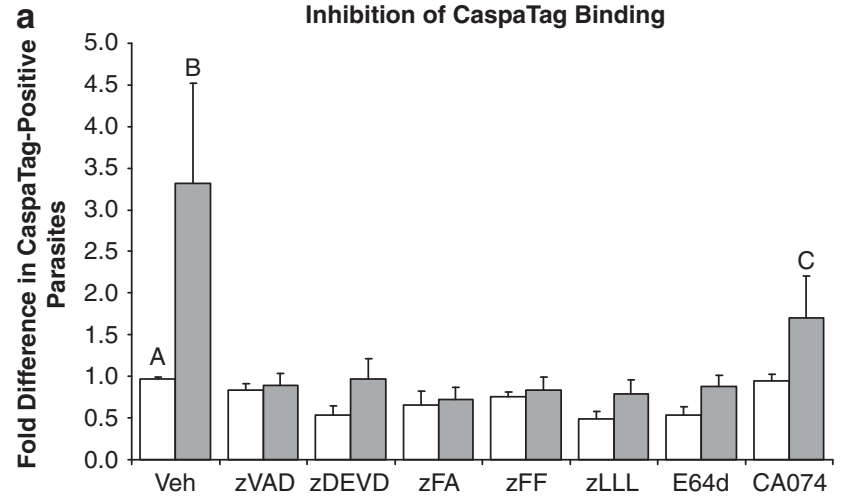

b

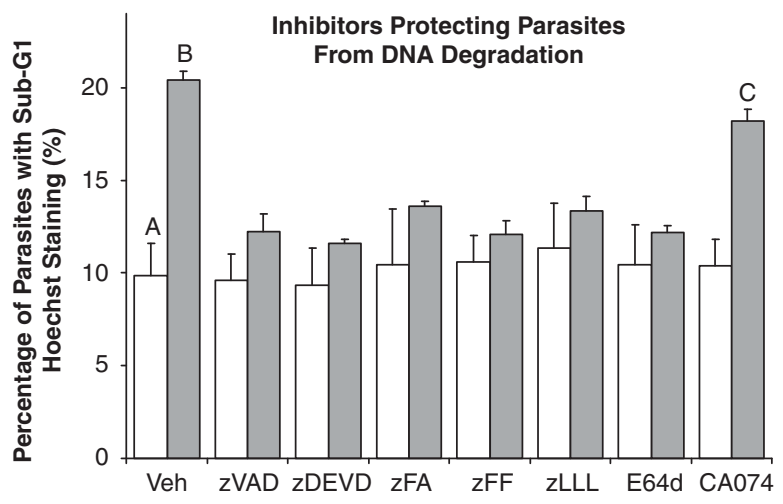

Figure 6 (a) CaspaTag staining of chloroquine-induced Plasmodium falciparum parasites pretreated with inhibitors. Percentage of CaspaTag-positive cells preincubated for $30 \mathrm{~min}$ with the following: Vehicle control; $50 \mu \mathrm{M}$ zVAD.fmk, zDEVD.fmk and CA-074; $5 \mu \mathrm{M}$ of zFA.fmk, zFF.fmk and zLLL.fmk; $12.5 \mu \mathrm{M}$ of E64d. Thereafter, cells were subjected to 8 -h treatment with vehicle control $\square$, $30 \mu \mathrm{M}$ chloroquine $\square$. Data represent means \pm S.E. (AvB, $P<0.001$; BvC, $P<0.01 ; A v C, P=0.054 ; N=3$ ) (b) Hoechst staining of parasites showing sub-G1 DNA content. The proportion of cells with weak Hoechst staining was assayed for $10 \mathrm{~h}$ after treatment with $\square$ or without $\square 30 \mu \mathrm{M}$ chloroquine. Cultures had been pretreated for $30 \mathrm{~min}$ with the following: Vehicle control; $50 \mu \mathrm{M}$ zVAD.fmk, zDEVD.fmk and CA-074; $5 \mu \mathrm{M}$ of zFA.fmk, zFF.fmk and zLLL.fmk; $12.5 \mu \mathrm{M}$ of E64d. Weak Hoechst staining was indicative of DNA degradation corresponding to a loss of DNA content leading to the sub-G1 population. Data represent means \pm S.E. (AvB and AvC, $P<0.001 ; \mathrm{BvC}, P<0.05 ; N=3$ )

Several established MOMP inhibitors were investigated (data not shown) and $4 \mathrm{HT}$ was shown to inhibit MOMP in CQ-induced cells without significantly reducing the proportion of healthy cells in the control group. Current evidence suggests that $4 \mathrm{HT}$ inhibits $\mathrm{Ca}^{2+}$-induced MOMP in ischemic myocardiocytes by binding to $\mathrm{Ca}^{2+}$-activated $\mathrm{K}^{+}$channels of the mitochondria. ${ }^{24-25}$ However, this is not likely to be the mechanism of action in the parasite as preincubation of parasites with the $\mathrm{Ca}^{2+}$ chelator BAPTA-AM did not prevent MOMP. In addition to $4 \mathrm{HT}$, the effectiveness of ZVAD in inhibiting MOMP in CQ-induced cells points clearly toward the activity of cysteine proteases being upstream of MOMP.

Despite some toxicity, $4 \mathrm{HT}$ was also able to partially reduce the major proportion of CaspaTag-positive cells in CQ-treated cultures, suggesting that MOMP occurs upstream of the bulk of cysteine protease activation in CQ-treated cells. This conclusion was supported by time course experiments that showed MOMP occurring in a significant proportion of cells at
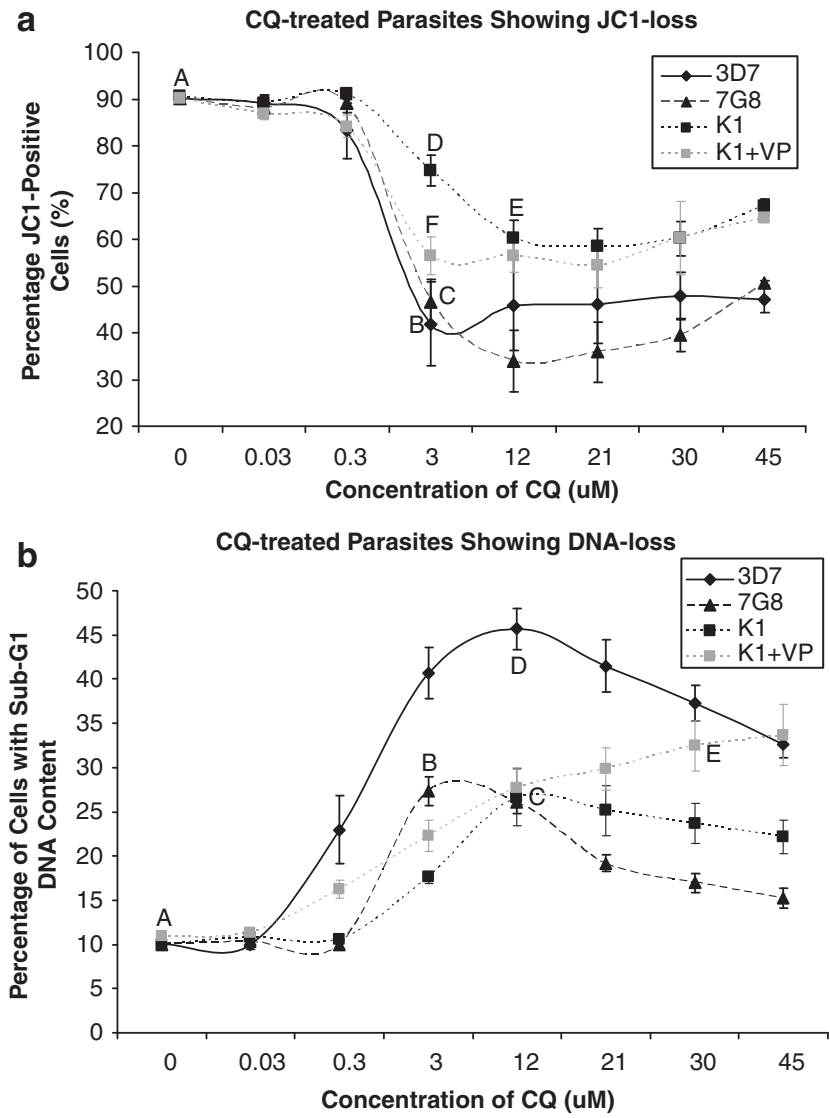

Figure 7 Drug-resistant parasites showing different PCD phenotype profiles. (a) Cultures of chloroquine-sensitive 3D7 and chloroquine-resistant 7G8 and K1 show different levels of JC1 loss at different concentrations of the drug. VP $+\mathrm{K} 1$ culture consisted of $\mathrm{K} 1$ parasites preincubated with $10 \mu \mathrm{M}$ of verapamil (VP) for $30 \mathrm{~min}$ before chloroquine treatments. Data represent means \pm S.E. (AvB and $A v C$, $P<0.001 ; \mathrm{BvD}$ and DvF, $P<0.01 ; \mathrm{AvE}, P<0.05 ; N=3$ ). (b) 3D7, $7 \mathrm{G} 8$ and $\mathrm{K} 1$ parasites showing different proportion of cells with sub-G1 DNA content as measured by Hoechst staining. Data represent means \pm S.E. (AvB, AvC, BvD and DvC, $P<0.001 ; \mathrm{DvE}, P<0.01 ; \mathrm{CvE}, P<0.05 ; N=3$ )

$4 \mathrm{~h}$ after $\mathrm{CQ}$ induction, whereas the increase in CaspaTagpositive cells was only significant after $6 \mathrm{~h}$.

Pretreatment with $4 \mathrm{HT}$ was unable to prevent $\mathrm{CQ}$-induced DNA fragmentation. Instead, $4 \mathrm{HT}$ toxicity made it difficult to interpret findings. In contrast, ZVAD pretreatment clearly reduced TUNEL staining in CQ-induced cells, placing the activation of cysteine proteases upstream of in situ DNA fragmentation.

The effectiveness of zVAD's inhibition of cysteine proteases, leading to a concomitant change in the proportion of CQ-induced parasites showing MOMP, cysteine protease activity and DNA fragmentation, is strongly indicative of a regulated pathway linking these $P C D$ features. In addition, the effect of $4 \mathrm{HT}$ in reducing CaspaTag-positive cells and time point experiments provides further insight into the $C Q$-induced pathway outlined below (Diagram 1b). Our model of CQ-induced PCD suggests that the activity of cysteine proteases is necessary for the loss of mitochondrial membrane potential. This preceding event is likely to be involved in 
a Classical Apoptotic Pathway:

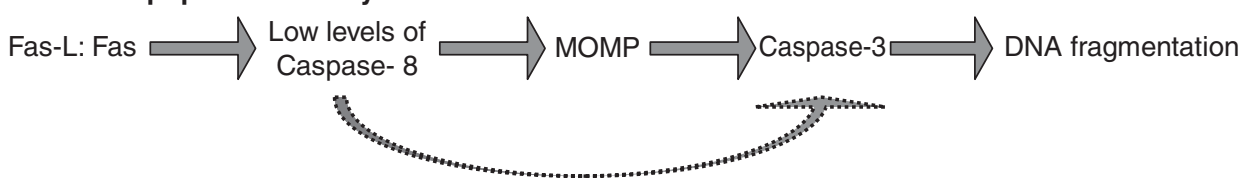

b Proposed PCD Model in 3D7 and CQ-resistant 7G8 and K1:

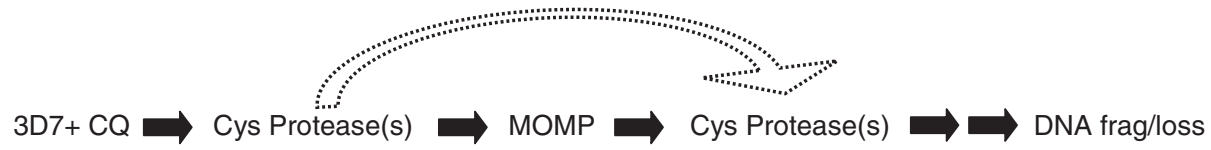

$7 \mathrm{G8}+\mathrm{CQ} \Rightarrow$ Cys Protease(s) $\Rightarrow$ MOMP $\Rightarrow$ Cys Protease(s) $\Rightarrow \square$ DNA frag/loss

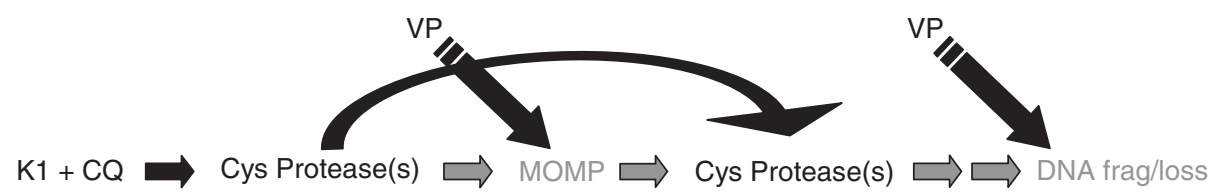

C Proposed Plasmodium falciparum Programmed Cell Death Model:

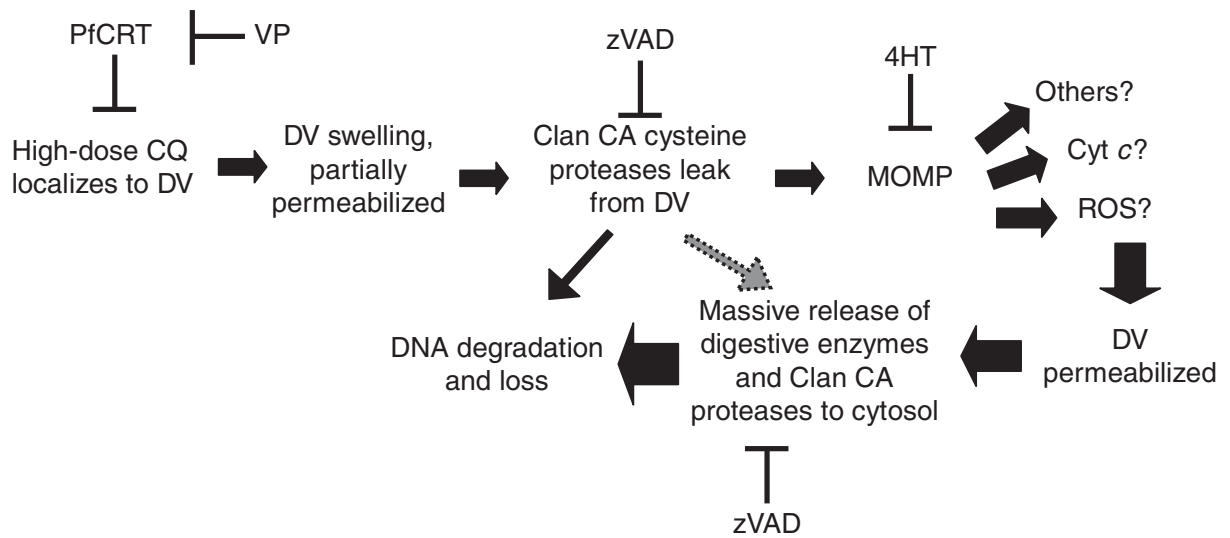

Diagram 1 (a) In the classical apoptotic pathway of mammalian cells, Fas-ligand binding to its receptor results in the activation of low levels of caspase 8 . Caspase 8 then results in a series of events leading to MOMP and the release of proapoptotic factors. These factors form the apoptosome with caspase 9 and elicit a strong caspase 3 response, ultimately inducing DNA fragmentation. (b) In 3D7 P. falciparum parasites treated with chloroquine (CQ), similar cell death features are observed. Inhibition studies with zVAD.fmk (ZVAD) and 4-hydroxytamoxifen (4HT) suggest that CQ treatment results in the activation of low levels of cysteine proteases, which triggers mitochondria outer membrane permeabilization (MOMP). In turn, this results in an increase in the amount of cysteine activity, which causes DNA degradation. This ordered sequence of events is characteristic of a programmed cell death pathway. This pathway is also likely to exist in CQ-resistant $7 \mathrm{G} 8$ parasites. Although showing similar proportions of cells with MOMP as 3D7, 7G8 parasites have a lesser degree of DNA degradation, suggesting that there are differences in the later-stage events of the pathway. In CQ-resistant K1 parasites, differences in both the degree of MOMP and DNA degradation suggest that the earlier stages of the pathway may differ from 3D7. The effectiveness of verapamil in reducing these differences shows the importance of CQ localization to the digestive vacuole (DV) in this pathway. Taken together with the results of other studies, a more detailed model of PCD in P. falciparum is proposed. (c) Only at high doses of $C Q$ does the drug accumulate in the DV to an extent that causes the DV to swell and become partially permeabilized. Clan CA proteases that are abundant in the DV leak into the cytoplasm of the parasite, resulting in the dysregulation of the mitochondrion. This places further stress on the DV leading to its rupture and a release of its contents (more clan CA proteases and other hydrolytic enzymes), which degrades the DNA and eventually the parasite

the amplification of protease activity, which results in DNA fragmentation. As experiments with $4 \mathrm{HT}$ were complicated by its toxicity, we cannot exclude the possibility that this amplification of the cysteine proteases can occur independently of mitochondrial permeabilization.

Our CQ-induced PCD model resembles the classical model of receptor-mediated apoptosis, which was recently reviewed by Degterev and Yuan. ${ }^{23}$ Although caspase 8 is able to directly activate executioner caspase 3 to initiate apoptosis, this process is greatly amplified through the mitochondrial route (Diagram 1a).

Although these models display overall similarities in the assayed characteristics, the absence of caspase homologs and classical mediators of apoptosis, such as Bid, Bax, AIF, 
Apaf-1 and Bcl-2 proteins, alludes to different mediators being involved in the process of $C Q$-induced PCD in $P$. falciparum. ${ }^{26}$

In ST-induced cells, the ineffectiveness of ZVAD and $4 \mathrm{HT}$ in reducing $P C D$ features suggests that a different phenomenon is being observed. The high concentrations of ST may have resulted in unregulated necrosis that is unlikely to be prevented by any number of inhibitors. ${ }^{27}$

An apparent limitation of this study is the high concentrations of $\mathrm{CQ}$ used. In an attempt to consolidate competing findings by different studies using different drug concentrations $(35 \mathrm{nM}-100 \mu \mathrm{M}),{ }^{6-8,19}$ parasites in this study were initially exposed to a range of drug concentrations (30, $300 \mathrm{nM}, 3$ and $30 \mu \mathrm{M}$ ) of $\mathrm{CQ}$ for $8 \mathrm{~h}$ and observed for features of PCD. To ensure that parasites were all still in a comparable trophozoite stage of development, an incubation time exceeding $10 \mathrm{~h}$ was not considered. As a significant loss of $\Delta \psi_{\mathrm{m}}$ and an increase in CaspaTag-positive cells were observed only with 3 and $30 \mu \mathrm{M}$ of $\mathrm{CQ}$ treatment, the latter dose was used. These parameters worked optimally with our protocols and allowed the investigation of multiple PCD phenotypes at a single time point. Moreover, effective zVAD-mediated inhibition of PCD features even at such high doses of $C Q$ suggests that key modulators of a well-regulated pathway are indeed being targeted.

Conclusions on studies using micromolar concentrations of CQ must not be projected as the primary mechanism of CQ's antimalarial activity. At physiological nanomolar concentrations, lysosomotropic $\mathrm{CQ}$ accumulates in the DV and interferes with hemazoin formation. ${ }^{28}$ However, our findings showed the drug being present in the cytoplasm of the parasite at micromolar concentrations where it could bind nonselectively to unknown cytosolic PCD mediators. Alternatively, relocalization of the drug at a high dose could be due to the excessive accumulation of the lysosomotropic drug in the DV leading to its compromise, the acidification of the cytoplasm and the accumulation of protonated $C Q$ inside the entire parasite.

To exclude the possibility that the proposed CQ-induced PCD pathway is an unregulated necrotic event, two necrosis controls were included. Both sodium azide and heat treatments resulted in distinctly different cell death profiles compared with CQ-induced cultures. Moreover, it was clearly shown that ZVAD was unable to modulate the extent of cell death features, strongly suggesting that these necrosis controls were genuinely undergoing an unregulated and disordered cell death. The ineffectiveness of zVAD in limiting necrotic cell death has been previously shown ${ }^{29-30}$ and contrasts with the well-ordered 'programmed' pathway characterized in $\mathrm{CQ}$-induced cells where downstream features of cell death can clearly be inhibited by $4 \mathrm{HT}$ and zVAD.

Although the concentration of the $C Q$ used limits this study's usefulness in understanding the therapeutic mechanisms of CQ's antimalarial activity, the objective of discovering novel mediators of $P$. falciparum PCD is not undermined. As further research characterizes what takes place in the parasite at such a dose of $C Q$, novel compounds specifically targeting the mediators of this PCD pathway may be optimally designed for antimalarial use.

Unlike the mammalian model, the identities of participating cysteine proteases in CQ-induced $P$. falciparum remains unknown, given the conspicuous absence of classical apoptotic mediators. Meslin et al. $^{8}$ have suggested that a clan CD protease PfMCA1 (a metacaspase homolog) was a likely mediator of cell death in $P$. falciparum. However, Le Chat et al. ${ }^{22}$ showed that $P$. berghei metacaspases did not have a role in PCD.

There is growing evidence that caspase inhibitors with fmk or aldehyde reactive groups (including CaspaTag) are not as specific as once believed, but can efficiently inhibit noncaspase cysteine proteases including metacaspases, calpains and cathepsins. ${ }^{10,14,17}$ This nonspecific nature of ZVAD binding in our study is confirmed by the fact that other fmk moiety-containing inhibitors with different amino-acid sequences (z-Asp-Glu-Val-Asp-fmk (zDEVD), zFA, zFF and $z L L L)$ seemed equally effective in reducing CaspaTag binding. Moreover, zFA, zFF and zLLL do not have the aspartate residue at the $\mathrm{P} 1$ position that is essential for classical caspase binding. Although the nonspecific nature of zVAD binding has allowed us to characterize the aforementioned pathway, we cannot ascertain the identity of its target protease on the basis of its binding to zVAD alone. Thus, clan CA-specific inhibitors such as E64d and CA-074 were included as well.

Despite being a broad-acting inhibitor of clan CA cysteine proteases, E64d does not crossreact with clan CD proteases. ${ }^{17,31-33}$ As E64d was effective at inhibiting CaspaTag binding and DNA loss in CQ-induced cells, we concluded that clan CA proteases are essential mediators of $C Q$-induced PCD. In addition, as CaspaTag binds both Clan CA and CD proteases, and Clan CA-specific E64d completely prevented the increase in CaspaTag-positive cells, this suggests that clan CA mediators are likely to be the proteases accounting for the cysteine protease activity being detected. The partial inhibition of PCD features by specific cathepsin B inhibitor, CA-074, shows that there may be multiple pathways involving clan CA proteases. Nevertheless, it would be premature to exclude the involvement of clan $C D$ proteases such as PfMCA1 as regulators or even key downstream mediators of CQ-induced PCD.

Conventionally, Clan CD caspases mediate apoptosis. However, recent work highlights the importance of clan CA papain-like proteases such as calpains and cathepsins in $P C D$ regulation through truncation of Bid or activation of $\mathrm{Bax}$, leading to MOMP. ${ }^{34-36}$ Even though Bid and Bax homologs do not exist in the parasite, it is conceivable that one or more parasite clan CA proteases may function likewise to cleave a novel parasite-specific mediator of MOMP. Clan CA proteases are well associated with parasites and include cathepsins and falcipains from the $\mathrm{C} 1$ family and calpains from the C2 family. ${ }^{17} P$. falciparum $3 \mathrm{D} 7$ possesses a preprocathepsin $C$ homolog, a calpain homolog, four falcipains, SERA (serine-repeat antigens) and other uncharacterized $\mathrm{C} 1$ proteases. ${ }^{18}$

Interestingly, a study by Gazarini et $a .^{37}$ showed that micromolar quantities of $C Q$ triggered calcium release from acidic compartments of $P$. chabaudi. Moreover, Farias et al. ${ }^{38}$ observed that calcium release was accompanied by a detection of cysteine-protease activity in the cytoplasm of $P$. chabaudi and $P$. falciparum. Although the authors suggest calpain involvement, this is unlikely as putative calpain 
homologs in Plasmodium do not have a calcium-binding domain. ${ }^{14}$ Our own studies on 3D7 also showed a relocalization of $\mathrm{Ca}^{2+}$ from the DV of the parasite to the cytoplasm. However, the use of the calcium chelator, BAPTA-AM, did not inhibit MOMP in CQ-induced parasites. However, the role of $\mathrm{Ca}^{2+}$ in limiting other features of $C Q$-induced $P C D$ has yet to be fully investigated and it is still possible that in $C Q$-induced PCD, cysteine protease are activated in a $\mathrm{Ca}^{2+}$-dependent mechanism as these authors have suggested.

This CQ-induced PCD pathway would only be of interest to drug developers if its existence were also established in CQ-resistant strains of $P$. falciparum. As such, drug-resistant $7 \mathrm{G} 8$ and $\mathrm{K} 1$ were also investigated for features of PCD. CQ resistance was initially believed to arise from mutations in the DV transmembrane protein CQ-resistance transporter (PfCRT). ${ }^{39}$ Two distinct sets of mutations were found, one originating from South America and the other from Southeast Asia and Africa. These mutations in PfCRT allow the efflux of $\mathrm{CQ}$ from its primary site of action, the DV, thereby conferring resistance to the drug. Interestingly, this resistance, originating from the Asia-Africa mutations, was shown to be reversible by VP and similarly structured compounds. ${ }^{40}$

Despite the differences between $7 \mathrm{G} 8$ and $\mathrm{K} 1$ in PfCRT mutation sets, both strains showed a significant proportion of cells with JC1-1 loss and DNA degradation, suggesting that PCD pathways exist even in these resistant strains. Interestingly, 7G8 showed a similar extent of cells exhibiting MOMP as 3D7 but a lower proportion of cells with sub-G1 content, even at higher $\mathrm{CQ}$ concentrations. In comparison, $\mathrm{K} 1$ showed a VP-reversible reduction in the proportion of cells with MOMP and DNA degradation that was only detectable at higher $C Q$ levels. These findings show the importance of PfCRT and CQ's localization in the DV for PCD to occur (Diagram 1b). In addition, the lesser extent of PCD features even at high $C Q$ concentrations suggests that these $C Q$-resistant strains may have a reduced propensity to undergo PCD. This model for drug resistance has also been established in cancer cells where treatment pressure leads to a selection of clones with altered apoptotic machinery or regulatory components. ${ }^{41}$ Such cells are more likely to survive and may be crossresistant to other drugs using the apoptotic pathway. Although further work on field isolates and other PCD inducers are needed, the potential of finding a link between drug resistance and resistance to cell death in $P$. falciparum would be very illuminating.

In summary, our present study not only shows that $\mathrm{CQ}$ and ST treatments give rise to characteristic features of PCD, but also a linear pathway implicating mitochondrial dysregulation, clan CA cysteine protease activity and DNA fragmentation is evident after $C Q$-induction. Although no specific mediators have been identified at this juncture, we have determined clan CA proteases as key pathway modulators. In addition, we have shown that PCD features exist in drug-resistant $7 \mathrm{G} 8$ and K1 parasites, and are suitable for therapeutic exploits. Besides providing promising targets for therapeutic drugs, breakthroughs in this area of PCD modulators should also enable a greater understanding of drug resistance and the physiological role of PCD pathways in unicellular organisms.

\section{Materials and methods}

Parasite culture. A CQ-sensitive laboratory strain of $P$. falciparum, 3D7 (MRA-102, MR4, ATCC, Manassas, Va, USA), and CQ-resistant strains 7G8 (MRA154, MR4, ATCC) and K1 (MRA-159, MR4, ATCC) were cultured continuously with minor modifications to the method previously described. ${ }^{42}$ Parasite cultures were maintained in 75 or $125 \mathrm{~cm}^{2}$ flasks using malaria culture media (MCM) consisting of RPMI 1640 supplemented with $0.5 \%$ (w/v) albumax I (Invitrogen, Auckland, New Zealand), $0.005 \%$ (w/v) hypoxanthine, $0.03 \%$ (w/v) L-glutamate, $0.25 \%$ (w/v) gentamycin and $2.5 \%$ human erythrocytes. Flasks were gassed with $3 \% \mathrm{CO}_{2}, 4 \% \mathrm{O}_{2}$ and $93 \% \mathrm{~N}_{2}$ and incubated at $37^{\circ} \mathrm{C}$ in a dark incubator. Culture medium was replenished during subculturing on alternate days. Thin Giemsa smears were made to determine parasitemia before subculture and at the start of each experiment. Sorbitol synchronization was carried out weekly to select for ring-stage parasites. ${ }^{43}$ Saponin enrichment was carried out before staining with CaspaTag and TUNEL assay to liberate the parasites from host erythrocytes. ${ }^{44}$ Cultures of approximately $7-10 \%$ parasitemia were used, with parasites being in the late-ring stage (22-26 h post-invasion) during the administration of inhibitor and drugs, unless otherwise stated.

Drug preparation and treatments. $C Q$ diphosphate (Sigma-Aldrich, Dorset, UK) was dissolved in PBS and filter-sterilized to obtain working solutions of $300 \mu \mathrm{M}$, and stored in the dark at $4^{\circ} \mathrm{C}$. A new batch of $\mathrm{CQ}$ working solution was made before each experiment. Unless otherwise stated, parasitized erythrocytes were incubated for 8 or $10 \mathrm{~h}$ in $30 \mu \mathrm{M}$ of $\mathrm{CQ}$ and washed twice with MCM before assaying for apoptotic features. Staurosporine (ST, Sigma-Aldrich) stock solution $(1 \mathrm{mM})$ was prepared by dissolving the drug in filtered dimethylsulfoxide (DMSO) and stored at $-20^{\circ} \mathrm{C}$. Working concentrations of ST at $100 \mu \mathrm{M}$ were prepared before each experiment by diluting the stock solution with MCM. Infected erythrocytes were treated for 8 or $10 \mathrm{~h}$ with $10 \mu \mathrm{M} \mathrm{ST}$, and washed twice with MCM before cell death assays. For necrosis controls, cultures were incubated with either $0.1 \%(\mathrm{w} / \mathrm{v})$ of sodium azide for 8 or $10 \mathrm{~h}$, or heat treated at $50^{\circ} \mathrm{C}$ for $1 \mathrm{~h}$, and then incubated at $37^{\circ} \mathrm{C}$ for 8 or $10 \mathrm{~h}$. Verapamil (VP, Sigma-Aldrich) was dissolved in PBS with $0.5 \%$ acetic acid, to a working concentration of $1 \mathrm{mM}$, and parasite cultures were pretreated with $10 \mu \mathrm{M}$ of VP for $30 \mathrm{~min}$ before adding CQ. Vehicle controls consisted of equivalent amounts of DMSO or acetic acid.

Synthesis of CM-CQ. Under a standard synthetic procedure, ${ }^{45}$ using flamedried glass apparatus under nitrogen atmosphere, $\operatorname{des}(N$-ethyl)-CQ $(25.0 \mathrm{mg}$, $0.09 \mathrm{mmol})$, that is, $N$-(7-chloro-4-quinolinyl)- $N^{\prime}$-ethyl-1,4-diaminobutane, ${ }^{46}$ was dissolved in anhydrous acetonitrile $(5.0 \mathrm{ml})$. Finely powdered and predried potassium carbonate $(24.8 \mathrm{mg}, 0.2 \mathrm{mmol})$ was then added, followed by the addition of an anhydrous acetonitrile solution $(1.0 \mathrm{ml})$ of the known $\mathrm{CM}$ $\alpha$-bromoester $(44.2 \mathrm{mg}, 0.1 \mathrm{mmol})$, that is, 2-bromo- $\mathrm{N}$-(2-(2-(7-(dimethylamino)-2oxo-2H-chromen-4-yl)acetamido)ethyl)-acetamide. ${ }^{16}$ The mixture was stirred for $13 \mathrm{~h}$ at room temperature, then filtered and the filtrate evaporated to give a yellow residue. The residue was then purified by flash column chromatography (230-400 mesh silica gel) to give CM-CQ ( $45.0 \mathrm{mg}, 0.074 \mathrm{mmol})$ in $82 \%$ yield.

NMR measurements of $\mathrm{CM}-\mathrm{CQ}$ were recorded on a $300 \mathrm{MHz}$ (for ${ }^{1} \mathrm{H}$ ) and $500 \mathrm{MHz}$ (for ${ }^{13} \mathrm{C}$ ) Bruker spectrometer (Fallanden, Switzerland) fitted with a pulse-field gradient probe. The residual resonance of deuterated solvents was used as an internal reference. ${ }^{1} \mathrm{H} \mathrm{NMR}(300 \mathrm{MHz}, \mathrm{MeOD}) \delta: 0.87(\mathrm{t}, J=6.4 \mathrm{~Hz}, 3 \mathrm{H}), 1.52-1.57(\mathrm{~m}, 2 \mathrm{H}), 1.62-1.74$ (m, 2H), 2.41 (t, $J=7.0 \mathrm{~Hz}, 2 \mathrm{H}$ ), 2.49 (q, $J=7.0 \mathrm{~Hz}, 2 \mathrm{H}$ ), 2.97 (bs, $8 \mathrm{H}), 3.21$ $(\mathrm{m}, 2 \mathrm{H}), 3.38-3.40(\mathrm{~m}, 4 \mathrm{H}), 3.63(\mathrm{~s}, 2 \mathrm{H}), 5.98(\mathrm{~s}, 1 \mathrm{H}), 6.34(\mathrm{~d}, J=2.3 \mathrm{~Hz}, 1 \mathrm{H}), 6.43$ $(\mathrm{d}, J=5.8 \mathrm{~Hz}, 1 \mathrm{H}), 6.55$ (dd, $J=2.6 \mathrm{~Hz}, 9.1 \mathrm{~Hz}, 1 \mathrm{H}), 7.25(\mathrm{dd}, J=2.0 \mathrm{~Hz}, 8.8 \mathrm{~Hz}$, $1 \mathrm{H}), 7.42(\mathrm{~d}, J=8.8 \mathrm{~Hz}, 1 \mathrm{H}), 7.71(\mathrm{~d}, J=2.0 \mathrm{~Hz}, 1 \mathrm{H}), 7.99(\mathrm{~d}, J=9.1 \mathrm{~Hz}, 1 \mathrm{H}), 8.29$ (d, $J=5.8 \mathrm{~Hz}, 1 \mathrm{H}$ ). ${ }^{13} \mathrm{C}$ NMR $(125 \mathrm{MHz}, \mathrm{MeOD}): \delta 12.1,26.2,27.1,40.0,40.1$, 40.2, 44.0, 47.4, 50.0, 55.7, 58.7, 98.6, 99.6, 109.7, 110.3, 110.5, 118.7, 124.5, 125.8, 126.8, 127.3, 136.2, 149.4, 152.2, 152.7, 154.6, 157.1, 164.2, 171.5, 175.2.

Analytical HPLC and mass spectrometry of CM-CQ was carried out on a Shimadzu LC-IT-TOF system (Kyoto, Japan) equipped with an autosampler, using

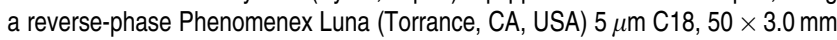
column. Solvent mixtures of $0.1 \% \mathrm{TFA} / \mathrm{H}_{2} \mathrm{O}$ (solvent-A) and $0.1 \% \mathrm{TFA} / \mathrm{CH}_{3} \mathrm{CN}$ (solvent-B) were used as coeluents under a linear gradient of $1-100 \%$ of solvent $\mathrm{B}$ (over $45 \mathrm{~min}$; flow rate of $0.5 \mathrm{ml} / \mathrm{min}$ ). This gave a purity of CM-CQ of $98 \%$ with a found $m / z[M+1]$ of 607.277 (calculated for $\mathrm{C}_{32} \mathrm{H}_{39} \mathrm{CIN}_{6} \mathrm{O}_{4}: 607.280$ ).

Inhibitor preparation and treatments. Inhibitors were all purchased from Sigma-Aldrich, dissolved in manufacturer's recommended solvents to stock concentrations and stored at $-20^{\circ} \mathrm{C}$. Working solutions were obtained by diluting the stock concentration with MCM just before administration. Parasite cultures were 
preincubated with inhibitors for $30 \mathrm{~min}$ before drug treatment. The final inhibitor concentrations were as follows: $4 \mathrm{HT}, 20 \mu \mathrm{M} ; \mathrm{zVAD}, \mathrm{zDEVD}$ and CA-074, $50 \mu \mathrm{M}$; zFA, zFF and zLLL, $5 \mu \mathrm{M}$; E64d, $12.5 \mu \mathrm{M}$; BAPTA-AM, 1-50 $\mu \mathrm{M}$. Lower concentrations of zFA, zFF and ZLLL were used because of their poor solubility. Vehicle control consisted of equivalent amounts of DMSO and/or ethanol in accordance with solvent amounts used in various inhibitor treatments.

Mitochondria transmembrane potential assay. Cell-permeable lipophilic cation probe JC-1 (Molecular Probes, Eugene, OR, USA), also known as 5,5', 6,6'tetrachloro-1,1',3,3'-tetraethyl-benzimidazolylcarbocyanine iodine, normally has green fluorescence $(525 \mathrm{~nm})$ but aggregates at high transmembrane potential of functional mitochondria to emit an orange-red fluorescence (excitation maximum at $590 \mathrm{~nm}){ }^{47}$ A final concentration of $6 \mu \mathrm{M}$ of $\mathrm{JC}-1$ was added to $0.5 \mathrm{ml}$ of treated parasitized erythrocytes and incubated at $37^{\circ} \mathrm{C}$ in a dark humidified candle jar for $30 \mathrm{~min}$. Cells were washed twice with PBS and resuspended to the original volume before being analyzed by flow cytometry. Percentage of JC1(Red)/JC1(Green) ratios were obtained to determine the extent of mitochondrial dysregulation as per the manufacturer's recommendations.

Cysteine protease activity assay. CaspaTag pancaspase in situ assay kit, sulforhodamine (Chemicon, Temecula, CA, USA), consists of a membrane-permeable fluoromethyl ketone inhibitor of caspases conjugated to sulforhodamine (SR-VAD-fmk). Saponin enrichment was performed on $2 \mathrm{ml}$ of treated cells, washed twice with $2 \mathrm{ml}$ PBS and resuspended in $300 \mu \mathrm{l} \mathrm{MCM}$. In accordance with the manufacturer's instructions, each vial of CaspaTag was dissolved in $50 \mu \mathrm{l}$ DMSO to produce the CaspaTag stock solution $(150 \times)$ and stored in the dark at $-20^{\circ} \mathrm{C}$ Before staining, further dilutions were made using MCM till a working solution of $30 \times$ CaspaTag was obtained. To each sample, $7 \mu \mathrm{l}$ of the freshly prepared CaspaTag reagent was added and the culture incubated at $37^{\circ} \mathrm{C}$ in the dark for $1 \mathrm{~h}$. Thereafter, two washes were carried out with $1 \mathrm{ml}$ of the wash buffer provided. The samples were resuspended in $1 \mathrm{ml}$ of wash buffer before flow cytometry analysis.

In situ DNA fragmentation assay. ApoDirect DNA Fragmentation Assay kit (Clontech, San Diego, CA, USA) is a TUNEL (terminal deoxynucleotidyltransferase dUTP nicked-end labeling) assay that makes use of FITC-conjugated dUTP incorporation into nicked DNA by means of terminal deoxynucleotidyltransferase. Saponin enrichment of $2 \mathrm{ml}$ of the treated cells also permeabilized the parasite's plasma membrane to the contents of the TUNEL stain. Parasites were then spun down and washed twice with $1 \mathrm{ml}$ of wash buffer provided in the kit. Samples were then resuspended in $50 \mu \mathrm{l}$ of the premixed staining solution, as directed by the manufacturer, and incubated in the dark for $90 \mathrm{~min}$ at $37^{\circ} \mathrm{C}$. Samples were then washed twice in $1 \mathrm{ml}$ of rinse buffer and resuspended in $1 \mathrm{ml}$ of PBS before flow cytometry.

Hoechst staining of parasite DNA content. Hoechst 33342 stain (provided together with CaspaTag kit, Chemicon) is a lipophilic DNA-binding fluorescent stain that is excited by ultraviolet light $(350 \mathrm{~nm})$, and emits blue fluorescence light $(461 \mathrm{~nm})$. After the parasite cultures were incubated in the absence/presence of drugs/inhibitors for $10 \mathrm{~h}$, cells were washed twice with PBS and $2 \mu \mathrm{l}$ of the $200 \mu \mathrm{g} / \mathrm{ml}$ stain was added per $1 \mathrm{ml}$ of culture for $30 \mathrm{~min}$. The cells were then washed twice and resuspended in PBS before flow cytometry.

Intracellular calcium detection. Cell-permeable Fluo-4-AM (Molecular Probes) fluoresces green when bound with $\mathrm{Ca}^{2+}$ and was used to detect intracellular calcium stores. A final concentration of $2 \mu \mathrm{M}$ of FLuo-4-AM was added to $0.5 \mathrm{ml}$ of treated parasitized erythrocytes and incubated at $37^{\circ} \mathrm{C}$ in a dark humidified candle jar for $30 \mathrm{~min}$. Cells were washed twice with PBS and resuspended to the original volume before viewing under a confocal microscope. At least 30 parasitized cells were counted for each condition.

Inhibitory concentration $\left(\mathbf{I C}_{50}\right)$ determination. Parasitized cultures (ring stage) diluted with fresh erythrocytes and MCM to $2 \%$ parasitemia and $1.25 \%$ hematocrit were incubated with various concentrations of commercial CQ (Sigma, Dorset, UK), CM (i.e., 7-dimethylamino-4-coumarinacetic acid) and $\mathrm{CM}-\mathrm{CQ}$ for $48 \mathrm{~h}$. To determine parasitemia, cultures were stained with Hoechst 33342 , as described above, and analyzed by flow cytometry.

Flow cytometric analyses. Cell numbers and fluorescence intensity after staining with JC-1, CaspaTag, FITC-dUTP or Hoechst were assayed by a flow cytometer (DAKO Cytomation Cyan ADP, Fort Collins, CO, USA), equipped with an argon-ion laser tuned to $488 \mathrm{~nm}$ and a violet laser tuned to $405 \mathrm{~nm}$. Blue, green and red fluorescence was detected using 450, 525 and $595 \mathrm{~nm}$ band-pass filters, respectively. For experiments involving $7 \mathrm{G8}$ and K1, a high-throughput flow cytometer (BD LSRII Special Order System, San Jose, CA, USA) was used to manage the large number of samples involved. Using BD LSR II, JC-1 stain was excited with $488 \mathrm{~nm}$ blue laser with green fluorescence being detected using the 505LP 525/50BP filter, whereas red fluorescence was detected using the 570LP 585/42BP filter. For Hoechst stain, excitation was by the $355 \mathrm{~nm}$ UV laser, whereas detection used the 450LP 450/ $50 \mathrm{BP}$ filter. Both flow cytometers were adjusted for forward and side scattering to accommodate either infected erythrocytes or liberated parasites. Between 100000 and 200000 erythrocytes or 10000-20000 enriched parasites were analyzed from each sample and its respective duplicate to ensure reliability. At least three independent experiments were performed unless otherwise stated.

Fluorescence and confocal microscopy. For fluorescence microscopy, wet mounts of stained infected erythrocytes or enriched parasites were observed using an image analysis setup consisting of a light and fluorescence microscope (Olympus BX60, Japan) coupled to a microscope digital camera (Olympus DP71, Tokyo, Japan) and images were captured using computer software DP Manager version 3.1.1.208 (Olympus). Confocal imaging was performed using Olympus Fluoview FV1000 (Japan) equipped with solid state and argon ion lasers tuned to 405 and $488 \mathrm{~nm}$, respectively. Images were captured using Olympus Fluoview version $1.6 \mathrm{~b}$. For enumeration purposes, images of at least 50 parasitized erythrocytes were screened per concentration of drug used.

Statistical analyses. All data shown are means \pm S.E.M. Statistical difference between percentages of stained cells was measured using univariate $t$-test for means and comparisons between multiple groups were analyzed using ANOVA and post hoc comparison using Tukey's test for paired comparisons. Significantly different results $(P<0.05)$ were highlighted.

\section{Conflict of interest}

The authors declare no conflict of interest.

Acknowledgements. We greatly appreciate the contributions of Jillian Cheong during the early stages of this project, Cynthia He for help with confocal microscopy, Georges Snounou and Laurent Renia for a critical reading of the manuscript, and George Cross for critical input on experimental design. Research from Kevin Tan's laboratory has been generously supported by a grant from the Infectious Disease Program, and Martin J. Lear gratefully acknowledges the Ministry of Education of Singapore for funding (AcRF Tier-2 Grant T206B1112). The use of the flow cytometry facility of the Singapore MIT Alliance for Research and Technology (SMART-ID IRG) is appreciated. C.J.H and S.R.K thank the National University of Singapore for Graduate Scholarships. Also, we thank MR4 for providing us with malaria parasites contributed by Daniel Carucci and Alister Craif (3D7) and Dennis Kyle (7G8 and K1).

1. World Malaria Report 2008. World Health Organization: Geneva, Switzerland, 2008.

2. White NJ. The role of anti-malarial drugs in eliminating malaria. Malar $J$ 2008; 7 (Suppl 1): S8.

3. Deponte M. Programmed cell death in protists. Biochim Biophys Acta 2008; 1783: 1396-1405.

4. Hurd H, Carter V. The role of programmed cell death in Plasmodium-mosquito interactions Int J Parasitol 2004; 34: 1459-1472.

5. Al-Olayan EM, Williams GT, Hurd H. Apoptosis in the malaria protozoan, Plasmodium berghei: a possible mechanism for limiting intensity of infection in the mosquito. Int $\mathrm{J}$ Parasitol 2002; 32: 1133-1143.

6. Nyakeriga AM, Perlmann H, Hagstedt M, Berzins K, Troye-Blomberg M, Zhivotovsky B et al. Drug-induced death of the asexual blood stages of Plasmodium falciparum occurs without typical signs of apoptosis. Microbes Infect 2006; 8: 1560-1568.

7. Totino PR, Daniel-Ribeiro CT, Corte-Real S, de Fatima Ferreira-da-Cruz M. Plasmodium falciparum: erythrocytic stages die by autophagic-like cell death under drug pressure. Exp Parasitol 2008; 118: 478-486.

8. Meslin B, Barnadas C, Boni V, Latour C, De Monbrison F, Kaiser $\mathrm{K}$ et al. Features of apoptosis in Plasmodium falciparum erythrocytic stage through a putative role of PfMCA1 metacaspase-like protein. $J$ Infect Dis 2007; 195: 1852-1859.

9. Kumar S, Guha M, Choubey V, Maity P, Srivastava K, Puri SK et al. Bilirubin inhibits Plasmodium falciparum growth through the generation of reactive oxygen species. Free Radic Biol Med 2008; 44: 602-613. 
10. Rozman-Pungercar J, Kopitar-Jerala N, Bogyo M, Turk D, Vasiljeva O, Stefe I et al. Inhibition of papain-like cysteine proteases and legumain by caspase-specific inhibitors: when reaction mechanism is more important than specificity. Cell Death Differ 2003; 10: 881-888.

11. Chauvier D, Ankri S, Charriaut-Marlangue C, Casimir R, Jacotot E. Broad-spectrum caspase inhibitors: from myth to reality? Cell Death Differ 2007; 14: 387-391.

12. Stoka V, Turk B, Schendel SL, Kim TH, Cirman T, Snipas SJ et al. Lysosomal protease pathways to apoptosis. Cleavage of bid, not pro-caspases, is the most likely route. J Biol Chem 2001; 276: 3149-3157.

13. Madeo F, Herker E, Maldener C, Wissing S, Lachelt S, Herlan M et al. A caspase-related protease regulates apoptosis in yeast. Mol Cell 2002; 9: 911-917.

14. Hurd H, Grant KM, Arambage SC. Apoptosis-like death as a feature of malaria infection in mosquitoes. Parasitology 2006; 132 (Suppl): S33-S47.

15. Turk B, Stoka V, Rozman-Pungercar J, Cirman T, Droga-Mazovec G, Oresic K et al. Apoptotic pathways: involvement of lysosomal proteases. Biol Chem 2002; 383: 1035-1044.

16. Alexander MD, Burkart MD, Leonard MS, Portonovo P, Liang B, Ding XB et al. A centra strategy for converting natural products into fluorescent probes. Chembiochem 2006; 7 409-416.

17. Mottram JC, Helms MJ, Coombs GH, Sajid M. Clan CD cysteine peptidases of parasitic protozoa. Trends Parasitol 2003; 19: 182-187.

18. Wu Y, Wang X, Liu X, Wang Y. Data-mining approaches reveal hidden families of proteases in the genome of malaria parasite. Genome Res 2003; 13: 601-616.

19. Porter H, Gamette MJ, Cortes-Hernandez DG, Jensen JB. Asexual blood stages of Plasmodium falciparum exhibit signs of secondary necrosis, but not classical apoptosis after exposure to febrile temperature (40 C). J Parasitol 2008; 94: 473-480.

20. Oakley MS, Kumar S, Anantharaman V, Zheng H, Mahajan B, Haynes JD et al. Molecular factors and biochemical pathways induced by febrile temperature in intraerythrocytic Plasmodium falciparum parasites. Infect Immun 2007; 75: 2012-2025.

21. McMorran BJ, Marshall VM, de Graaf C, Drysdale KE, Shabbar M, Smyth GK et al. Platelets kill intraerythrocytic malarial parasites and mediate survival to infection. Science 2009; 323: 797-800

22. Le Chat L, Sinden RE, Dessens JT. The role of metacaspase 1 in Plasmodium berghei development and apoptosis. Mol Biochem Parasitol 2007; 153: 41-47.

23. Degterev A, Yuan J. Expansion and evolution of cell death programmes. Nat Rev Mol Cell Biol 2008: 9: 378-390.

24. Morkuniene R, Jekabsone A, Borutaite V. Estrogens prevent calcium-induced release of cytochrome $c$ from heart mitochondria. FEBS Lett 2002; 521: 53-56.

25. Ohya S, Kuwata Y, Sakamoto K, Muraki K, Imaizumi Y. Cardioprotective effects of estradio include the activation of large-conductance $\mathrm{Ca}(2+)$-activated $\mathrm{K}(+)$ channels in cardiac mitochondria. Am J Physiol Heart Circ Physiol 2005; 289: H1635-H1642.

26. Deponte M, Becker K. Plasmodium falciparum - do killers commit suicide? Trends Parasitol 2004; 20: 165-169.

27. Studzinski DM, Benjamins JA. Cyclic AMP differentiation of the oligodendroglial cell line N20.1 switches staurosporine-induced cell death from necrosis to apoptosis. J Neurosci Res 2001; 66: 691-697.

28. Goldberg DE, Slater AF, Cerami A, Henderson GB. Hemoglobin degradation in the malaria parasite Plasmodium falciparum: an ordered process in a unique organelle. Proc Natl Acad Sci USA 1990; 87: 2931-2935.

29. Kim SO Han J. Pan-caspase inhibitor ZVAD enhances cell death in RAW246.7 macrophages. J Endotoxin Res 2001; 7: 292-296.

30. Sperandio S, de Belle I, Bredesen DE. An alternative, nonapoptotic form of programmed cell death. Proc Natl Acad Sci USA 2000; 97: 14376-14381.
31. Tamai M, Yokoo C, Murata M, Oguma K, Sota K, Sato E et al. Efficient synthetic method for ethyl (+)-(2S,3S)-3-[(S)-3-methyl-1-(3-methylbutylcarbamoyl)butylcarbamoyl]-2-oxiranecarb oxylate (EST), a new inhibitor of cysteine proteinases. Chem Pharm Bull (Tokyo) 1987; 35: 1098-1104.

32. McGowan EB, Becker E, Detwiler TC. Inhibition of calpain in intact platelets by the thiol protease inhibitor E-64d. Biochem Biophys Res Commun 1989; 158: 432-435.

33. Nicholson DW, Ali A, Thornberry NA, Vaillancourt JP, Ding CK, Gallant M et al. Identification and inhibition of the ICE/CED-3 protease necessary for mammalian apoptosis. Nature 1995; 376: 37-43.

34. Broker LE, Kruyt FA, Giaccone G. Cell death independent of caspases: a review. Clin Cancer Res 2005; 11: 3155-3162.

35. Bursch $W$. The autophagosomal-lysosomal compartment in programmed cell death. Cell Death Differ 2001; 8: 569-581.

36. Borner C, Monney L. Apoptosis without caspases: an inefficient molecular quillotine? Cell Death Differ 1999; 6: 497-507.

37. Gazarini ML, Sigolo CA, Markus RP, Thomas AP, Garcia CR. Antimalarial drugs disrupt ion homeostasis in malarial parasites. Mem Inst Oswaldo Cruz 2007; 102: 329-334.

38. Farias SL, Gazarini ML, Melo RL, Hirata IY, Juliano MA, Juliano L et al. Cysteine-protease activity elicited by $\mathrm{Ca2}+$ stimulus in Plasmodium. Mol Biochem Parasitol 2005; 141: 71-79.

39. Fidock DA, Nomura T, Talley AK, Cooper RA, Dzekunov SM, Ferdig MT et al. Mutations in the $P$. falciparum digestive vacuole transmembrane protein PfCRT and evidence for their role in chloroquine resistance. Mol Cell 2000; 6: 861-871.

40. Yeh S, Smith PJ, Chibale K. Dual-acting diamine antiplasmodial and chloroquine resistance modulating agents. Biochem Pharmacol 2006; 72: 156-165.

41. Pommier Y, Sordet O, Antony S, Hayward RL, Kohn KW. Apoptosis defects and chemotherapy resistance: molecular interaction maps and networks. Oncogene 2004; 23: 2934-2949.

42. Trager W, Jensen JB. Human malaria parasites in continuous culture. 1976. J Parasito 2005; 91: 484-486.

43. Lambros $C$, Vanderberg JP. Synchronization of Plasmodium falciparum erythrocytic stages in culture. J Parasitol 1979; 65: 418-420.

44. Beaumelle BD, Vial HJ, Philippot JR. Reevaluation, using marker enzymes, of the ability of saponin and ammonium chloride to free Plasmodium from infected erythrocytes. J Parasitol 1987; 73: 743-748.

45. Prusov E, Maier ME. Synthesis of nitrogen-containing spirocyclic scaffolds via aminoallylation/RCM sequence. Tetrahedron 2007; 63: 10486-10496.

46. Natarajan JK, Alumasa JN, Yearick K, Ekoue-Kovi KA, Casabianca LB, de Dios AC et al. 4-N-, 4-S-, and 4-O-chloroquine analogues: influence of side chain length and quinolyl nitrogen pKa on activity vs chloroquine resistant malaria. J Med Chem 2008; 51: $3466-3479$.

47. Smiley ST, Reers M, Mottola-Hartshorn C, Lin M, Chen A, Smith TW et al. Intracellular heterogeneity in mitochondrial membrane potentials revealed by a J-aggregate-forming lipophilic cation JC-1. Proc Natl Acad Sci USA 1991; 88: 3671-3675.

Cell Death and Disease is an open-access journal published by Nature Publishing Group. This article is licensed under a Creative Commons Attribution-Noncommercial-No Derivative Works 3.0 License. To view a copy of this license, visit http:// creativecommons.org/licenses/by-nc-nd/3.0/

\section{Supplementary Information accompanies the paper on Cell Death and Disease website (http://www.nature.com/cddis)}

